\title{
Relaxation and breakup of an initially extended drop in an otherwise quiescent fluid
}

\author{
By H. A. STONE † AND L. G. LEAL \\ Department of Chemical Engineering, California Institute of Technology, \\ Pasadena, CA 91125, USA
}

(Received 30 September 1987 and in revised form 24 June 1988)

In this paper we examine some general features of the time-dependent dynamics of drop deformation and breakup at low Reynolds numbers. The first aspect of our study is a detailed numerical investigation of the 'end-pinching' behaviour reported in a previous experimental study. The numerics illustrate the effects of viscosity ratio and initial drop shape on the relaxation and/or breakup of highly elongated droplets in an otherwise quiescent fluid. In addition, the numerical procedure is used to study the simultaneous development of capillary-wave instabilities at the fluid-fluid interface of a very long, cylindrically shaped droplet with bulbous ends. Initially small disturbances evolve to finite amplitude and produce very regular drop breakup. The formation of satellite droplets, a nonlinear phenomenon, is also observed.

\section{Introduction}

The study of the low Reynolds number deformation and breakup of a liquid drop due to a non-uniform velocity field is a classical free-boundary problem which has been of longstanding interest in the fluid mechanics community, dating back to two remarkable papers by $\mathrm{G}$. I. Taylor in the $1930 \mathrm{~s}$.

Two distinct approaches have been taken in order to understand different aspects of the drop breakup problem. The first approach examines the deformation of an initially spherical droplet, with undeformed radius $a$, in sleady linear flows. The major objective of these studies is the critical capillary number $\mathbb{C}=G a \mu / \sigma$ ( $G$ is the local shear rate, $\mu$ is the suspending fluid viscosity, and $\sigma$ denotes the interfacial tension), which corresponds to the limit point for steady drop shapes (on the branch of solutions which begin with a sphere at $\mathbb{C}=0$ ). This work was summarized recently in review articles by Acrivos (1983) and Rallison (1984), though an important extension was presented by Bentley \& Leal (1986) who systematically investigated the inhibiting effect of vorticity on the deformation and breakup process, a feature first observed and explained by Taylor (1934). The term 'drop breakup', as used in these investigations, generally refers to the non-existence of a steady solution. However, actual fragmentation of the drop is not discussed in these studies.

For $\mathbb{C}$ larger than the critical value, the droplet stretches continuously to highly elongated shapes that have long cylindrical midsections with generally bulbous ends. These elongated shapes provide the motivation for the second major class of studies on drop breakup. In this case, the droplet is modelled as an infinite fluid cylinder of initially uniform radius which breaks up in quiescent or sheared flows due to the

$\dagger$ Present address: Division of Applied Sciences, Harvard University, Cambridge, MA 02138 USA. 
growth of capillary-wave instabilities. Within the confines of linear stability theory, the effects of viscosity ratio and disturbance wavelength on the disturbance growth rate and resulting drop size distribution are examined by Tomotika (1935), Rumscheidt \& Mason (1962) and Lee \& Flumerfelt (1981) for the case of quiescent fluids, and by Tomotika (1936), Mikami, Cox \& Mason (1975) and Khakhar \& Ottino (1987) for droplets in a shearing flow. For the equivalent problem where inertial effects rather than viscous effects are important, there have been many studies that examine nonlinear details of the interface evolution (for example, see the review article by Bogy 1979). However, as far as we are aware, nonlinear effects have not been studied for the Stokes flow problem.

While these previous studies have been very valuable in understanding the basic physics of flow-induced changes in the drop shape, and have yielded some insight into the factors that control drop size distributions on breakup, it is nevertheless true that real processes almost always involve time-dependent flows (as seen by the drop) and it is important to ask how transient effects may alter the deformation and breakup process. Furthermore, there remain many outstanding problems related to capillary wave growth on extended fluid drops. For example: what role does the finite length of the droplet play? how do finite-amplitude disturbances evolve? and how are nonlinear effects evident? These aspects of the drop deformation problem have received relatively little attention in the literature.

In a previous publication (Stone, Bentley \& Leal 1986) we took a first step towards better understanding of the time-dependent dynamics of modestly deformed drops. Specifically, we experimentally examined droplet stretching near the critical capillary number in two-dimensional linear flows generated in a computer-controlled four-roll mill and investigated the relaxation dynamics that occur if the imposed flow is stopped abruptly with the droplet in a stretched, non-equilibrium state. In the relaxation experiments it was frequently observed that breakup occurred due to deterministic flows established by capillary forces associated with curvature variations along the interface, rather than by a capillary-wave instability. The observed motion consisted of a relatively rapid bulbing of the end of the drop followed by break-off of the bulbous end from the central portion of the drop. This breakup process, called 'end-pinching', was shown to have a rather strong dependence on the ratio of drop viscosity to suspending fluid viscosity, $\lambda(\lambda=\hat{\mu} / \mu$ where $\hat{\mu}$ is the droplet viscosity). Specifically, for $\lambda>O(1)$, the mechanism leading to breakup was damped and much larger elongations were necessary to guarantee breakup as the viscosity ratio was increased. The effect of increasing the droplet extension prior to stopping the flow was also examined, and, for sufficiently long drops, capillary waves were shown to play an important role in the final stages of the breakup process. Finally, a qualitative explanation of the 'end-pinching' process was proposed and a brief supporting numerical computation presented for the special case $\lambda=1$.

In this paper we present more detailed numerical calculations using the boundaryintegral method to further elucidate the end-pinching mechanism for a highly extended droplet of constant interfacial tension suspended in an otherwise quiescent fluid. Our basic interest is to probe the effects of viscosity ratio and initial drop shape on the relaxation and breakup process, with a particular emphasis on understanding the end-pinching behaviour. We also use the numerical procedure to probe some (nonlinear) features associated with the growth of capillary-wave instabilities on a highly elongated droplet. Of course, an obvious and, from the standpoint of applications, important question is the role of surface-tension gradients in drop 
deformation and breakup due, for example, to the presence of surfactants at the fluid-fluid interface. This subject is currently under study and will be addressed in a future communication.

It is worth noting that the transient dynamics described in this paper are related to other free-boundary problems which arise in quite varied processes. Similar relaxation and breakup processes are illustrated by Lasheras, Fernandez-Pello \& Dryer (1979) in an experimental study of the combustion characteristics of fuel droplets and by Greenspan \& McCay (1981) for the retraction of an initially extended droplet that wets a solid planar surface. $\dagger$ Another closely related problem is the capillary instability of inviscid liquid jets, which has been widely studied owing to a variety of practical applications. For example, similar dynamics to aspects of this work are illustrated and discussed by Goedde \& Yuen (1970).

\section{Numerical procedure/implementation}

The primary objective of this study is to examine the details of relaxation and breakup of highly elongated droplets suspended in an otherwise quiescent fluid. A very efficient numerical scheme for this type of free-boundary problem is the boundary-integral method. The basic boundary-integral formulation is fairly widespread in the fluid-mechanics literature. For example, at low Reynolds number it has been used to study flow past solid particles by Youngren \& Acrivos (1975), the approach of a particle or drop towards a fluid-fluid interface by Leal and coworkers (Lee \& Leal 1982; Geller, Lee \& Leal 1986) and flow over two-dimensional slits by Higdon (1985).

The first application of the boundary-integral method to the drop deformation problem was due to Youngren \& Acrivos (1976) who studied bubble shapes in an axisymmetric extensional flow, and by Rallison \& Acrivos (1978) for viscous drops. Specifically, the latter authors calculate steady drop shapes for viscosity ratios $\lambda$ in the range $0.3<\lambda<100$. For the special case $\lambda=1.0$ the calculations were extended by Rallison (1981) to deformation in a simple shear flow and the method has also been applied to drop deformation in electric and magnetic fields by Sherwood (1988). The numerical procedure used in the present study incorporates ideas presented in the above-mentioned studies, in addition to those of $\mathrm{E}$. J. Hinch (private communication).

For the sake of completeness we summarize the necessary integral equations below. For more details, the reader is referred to Rallison \& Acrivos (1978). The formulation is given for the general situation with $\boldsymbol{u}_{\infty} \neq 0$, though, for the most part, it is applied in this paper only for the case of a quiescent far-field flow, $\boldsymbol{u}_{\infty}=0$. The dimensionless governing equations are the quasi-steady Stokes equation and continuity equation for each phase:

$$
\begin{array}{cc}
\nabla^{2} \boldsymbol{u}=\boldsymbol{\nabla} p, & \nabla^{2} \hat{\boldsymbol{u}}=\boldsymbol{\nabla} \hat{p} \\
\boldsymbol{\nabla} \cdot \boldsymbol{u}=0, & \boldsymbol{\nabla} \cdot \hat{\boldsymbol{u}}=\mathbf{0} .
\end{array}
$$

Variables associated with the droplet phase are denoted by the symbol ^. All velocities have been non-dimensionalized by $u_{\mathrm{c}}=\sigma / \mu$, time by the convective timescale $t_{\mathrm{c}}=l_{\mathrm{c}} / u_{\mathrm{c}}$ where $l_{\mathrm{c}}$ is a characteristic lengthscale, and pressures by $p_{\mathrm{c}}=\mu u_{\mathrm{c}} / l_{\mathrm{c}}, \hat{p}_{\mathrm{c}}=\hat{\mu} u_{\mathrm{c}} / l_{\mathrm{c}}$. The choice of characteristic velocity is appropriate for relaxation of initially elongated droplets in an otherwise quiescent fluid. For a nearly

+ We wish to thank these authors for bringing these studies to our attention. 
spherical drop the undeformed radius, $a$, is an appropriate lengthscale while for a highly extended drop the midsection radius is more appropriate.

The boundary conditions are

$$
\begin{aligned}
& u(x) \rightarrow u_{\infty} \text { as }|x| \rightarrow \infty, \\
& u=\hat{u} \text { for } x \in x_{s}, \\
& n \cdot T-\lambda \boldsymbol{n} \cdot \hat{\boldsymbol{T}}=n\left(\nabla_{s} \cdot \boldsymbol{n}\right) \text { for } x \in \boldsymbol{x}_{s}
\end{aligned}
$$

and the kinematic condition

$$
\frac{\mathrm{d} \boldsymbol{x}_{s}}{\mathrm{~d} t}=\boldsymbol{n}(\boldsymbol{u} \cdot \boldsymbol{n})
$$

describes the evolution of the drop shape. The position vector $x$ denotes a point in the fluid domain, while $\boldsymbol{x}_{s}$ indicates a point at the fluid-fluid interface. $\boldsymbol{T}$ and $\hat{\boldsymbol{T}}$ denote the stress tensors for the continuous and droplet phases respectively, $n$ is the unit normal directed from the droplet phase to the continuous phase and $\nabla_{8} \cdot \boldsymbol{n}$ represents the mean curvature of the fluid-fluid interface (see figure 1). Owing to our choice for $u_{\mathrm{c}}$, the capillary number, $\mathbb{C}=G a \mu / \sigma$ ( $G$ is the shear rate of the external flow $\boldsymbol{u}_{\infty}$ ) appears explicitly in the definition of $\boldsymbol{u}_{\infty}$ (see (4)), but does not appear in the stress balance at the interface. It is assumed, in the above equations and boundary conditions that the drop is neutrally buoyant, i.e. $\rho=\hat{\rho}$.

The complete neglect of inertia, upon which this study is predicated, requires that both local and convective acceleration effects are small compared to viscous effects. For relaxation phenomena when $\mathbb{C}=0$ this requires $\sigma \rho l_{\mathrm{c}} / \mu^{2}(1+\lambda) \ll 1(\rho$ is the density of the suspending fluid), which will generally be satisfied for small droplets suspended in viscous fluids. In the presence of an imposed linear flow, with characteristic velocity scale $G l_{\mathrm{c}}$, the neglect of inertia also requires that $\rho G l_{\mathrm{c}}^{2} / \mu \ll 1$. For time-dependent flows it is necessary to neglect inertial effects due to the transient nature of the flow. Local acceleration effects are negligible compared with viscous effects provided $\rho l_{\mathrm{c}}^{2} / \mu \tau \ll 1$, where $\tau$ is the timescale characterizing the transient flow.

In the boundary-integral formulation, the velocity at any point in the two phases is represented by

$$
\begin{gathered}
u(x)=u_{\infty}(x)-\int_{S} n \cdot \boldsymbol{T} \cdot J \mathrm{~d} S(y)-\int_{S} n \cdot \boldsymbol{K} \cdot \boldsymbol{u} \mathrm{d} S(y) \\
\hat{u}(x)=\int_{S} n \cdot \hat{\boldsymbol{T}} \cdot J \mathrm{~d} S(y)+\int_{S} \boldsymbol{n} \cdot \boldsymbol{K} \cdot \hat{u} \mathrm{~d} S(y)
\end{gathered}
$$

where

$$
\begin{aligned}
& J=\frac{1}{8 \pi}\left[\frac{I}{|x-y|}+\frac{(x-y)(x-y)}{|x-y|^{3}}\right], \\
& K=-\frac{3}{4 \pi} \frac{(x-y)(x-y)(x-y)}{|x-y|^{5}} .
\end{aligned}
$$

In these equations, $S$ represents the drop surface and $y$ is the integration variable. The pressure field can be similarly represented by surface integrals involving the velocity and stress fields at the interface. Hence, provided that the interfacial stress and interfacial velocity distributions are known, knowledge of the drop shape allows the velocity and pressure fields to be determined anywhere in the fluid domain.

Making use of the boundary conditions and the well-known jump conditions to treat the singular nature of the kernel in the limit $\boldsymbol{x} \rightarrow \boldsymbol{x}_{s} \in S$ the general equations (1) 


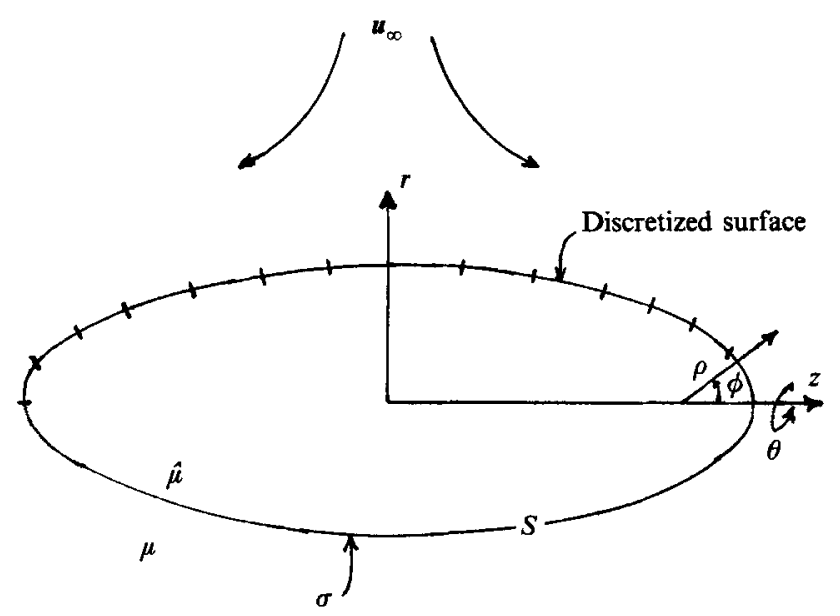

Figure 1. Definition of variables for a deformed droplet in an extensional flow.

and (2) lead to an integral equation for the interfacial velocity (Rallison \& Acrivos 1978)

$$
\frac{(1+\lambda)}{2} \hat{u}\left(x_{s}\right)=u_{\infty}\left(x_{s}\right)-\int_{S} \boldsymbol{J} \cdot \boldsymbol{n}\left(\nabla_{s} \cdot n\right) \mathrm{d} S(y)-(1-\lambda) \int_{S} n \cdot \boldsymbol{K} \cdot \hat{u} \mathrm{~d} S(y) .
$$

For a given shape, capillary number and viscosity ratio, this expression is an integral equation of the second kind for the interfacial $\hat{u}\left(x_{s}\right)$. As there exists no known analytic solution to this equation we must resort to an approximate numerical solution. The case $\lambda=1$ is especially straightforward, as is evident from (3).

In the interest of numerical simplicity, we consider an initially axisymmetric drop which then remains axisymmetric for all time. Referring to the cylindrical coordinate system shown in figure 1 , the shape is also symmetric with respect to a plane passing through $z=0$, orthogonal to the axial $(z)$ direction. In $(3)$ the azimuthal $(\theta)$ integration can be performed analytically and the surface integrals are reduced to line integrals. The resulting one-dimensional integral equation for the interfacial velocity field is solved by discretizing the interface into $2 N-2$ boundary elements with node points placed at the end of each element, and converting the integral equation to an 'equivalent' linear system of coupled algebraic equations by approximating the unknown function $\hat{u}\left(x_{s}\right)$ over each element of the surface. Owing to the axisymmetry, there are two unknown components of the interfacial velocity $\left(\hat{u}_{r}, \hat{u}_{z}\right)$ at each node point.

There are two important aspects to the discretization: (i) approximation of the variation of interfacial velocity over an element of the surface; and (ii) accurate representation of the drop shape and curvature. The former is treated by assuming that the velocity field varies linearly over each element. For the highly deformed shapes typical of this study, tests with this representation demonstrated that it helped to produce smoother and more accurate interfacial velocity distributions than the more common assumption of constant velocity over each element. Accurate representation of the drop shape is crucial simply because many of the motions we wish to study are driven solely by curvature variations over the drop surface. In order to generate a smooth and accurate representation of the interface without excessive use of collocation points two alternative methods are utilized.

Since many of the shapes to be studied have cylindrical midsections with bulbous, 
nearly spherical ends, the first scheme makes use of cylindrical coordinates near the middle of the drop and spherical coordinates near the end of the drop (the surface, with $2 N-1$ collocation node points, is described in these two cases by $r=r(z)$ and $\rho=\rho(\phi)$ respectively - see figure 1) with cubic splines used to generate twice continuously differentiable representations of the interface in each region. The two representations are patched together by requiring first derivatives to be continuous at the point of overlap and second derivatives to be continuous within some tolerance (typically $10^{-6}$ ) at the point of overlap. In this way the drop shape is described by a smooth function which is essentially twice continuously differentiable everywhere. The second scheme uses an arclength parametrization method (Ascoli 1987). If $s$ represents a normalized measure of arclength $(0 \leqslant s \leqslant 1)$, then the collocation points on the interface, labelled using the cylindrical coordinates $(r, z)$, are parametrized so that $r=r(s), z=z(s)$ describes the fluid-fluid interface and again twice continuously differentiable representations are generated using cubic splines. Both methods work very well, although for a similar number of points, as might be expected, the former scheme is more successful for elongated shapes with nearly spherical ends while the latter scheme performs better for very slender shapes with nearly pointed ends.

Owing to the fore-aft symmetry, the number of unknowns is halved and the net result of discretization is a system of $2 N-2$ equations and unknowns. The resulting linear system is solved using Gaussian elimination to give the interfacial velocity distribution along the fluid-fluid interface.

Typically, for relaxation of the extended drops illustrated in $\$ 3.2$, we choose $N=35$ for drops with initial half-lengths $30-40$ times the midsection radius. In a study of capillary instabilities on a highly elongated drop, described in $\S 3.3$, we choose $N=49$. In both studies, the collocation points initially have an average spacing of $\Delta z \approx 1.0-1.5$ in the cylindrical region and are more densely spaced near the bulbous end where larger curvature variations occur. With this node-point spacing the numerics are capable of resolving small initial disturbances on the cylindrical region whose frequencies $\omega$ are smaller than 2 . Since the drop shortens as the relaxation process proceeds (and, consequently, the average point spacing decreases), the same number of points is capable also of resolving the larger curvature variations which occur at later times, either due to the end-pinching phenomena or the growth of capillary instabilities. Limited testing in these cases with $50 \%$ more collocation elements yielded the same results.

In order to demonstrate the accuracy of the shape-fitting routine, we compare analytically determined curvatures, $\left(\nabla_{s} \cdot \boldsymbol{n}\right)$, with numerically generated values for an object of known shape. The test shape is shown in the inset to table 1 and consists of a uniform cylindrical midsection connected to bulbous ends. The end-to-end length of the drop is 106 times its undeformed radius. The dark symbols on the interface indicate the location of the node points. On the cylindrical midsection a sinusoidal disturbance, frequency $\omega$ and amplitude $R_{a}$, is superimposed. At each node point in the cylindrical region we calculate the relative error between the numerical and analytical values of the curvature (error $=$ (exact - numerical)/exact). In table 1 we display the maximum error and square root of the mean-square error. Also shown is the dominant frequency obtained from a Fourier analysis of the central cylindrical region (calculated using (5)). The cases $N=49$ have an average point spacing between collocation points in the cylindrical region of $\Delta z \approx 1.0$ and the cases $N=69$ have an average spacing of $\Delta z \approx 0.6$. Clearly, the numerical procedure does a good job of approximating the curvature and in all cases the Fourier spectrum has a sharp peak at the frequency of the applied disturbance. This test of the shape-fitting 


\begin{tabular}{|c|c|c|c|c|c|c|c|}
\hline & $N$ & $\begin{array}{l}\text { Number of } \\
\text { points on } \\
\text { midsection } \\
(0<z<30)\end{array}$ & $R_{a}$ & $\omega$ & $\begin{array}{c}\text { Maximum } \\
\text { error }\end{array}$ & $\begin{array}{c}\text { Square root of } \\
\text { mean square } \\
\text { error }\end{array}$ & $\begin{array}{c}\text { Fourier } \\
\text { decomposition } \\
\text { frequency }\end{array}$ \\
\hline $\begin{array}{r}1 a \\
b\end{array}$ & $\begin{array}{l}49 \\
69\end{array}$ & $\begin{array}{l}30 \\
50\end{array}$ & 0.2 & $\begin{array}{l}0.5 \\
-\end{array}$ & $\begin{array}{l}2.0 \times 10^{-3} \\
8.6 \times 10^{-4}\end{array}$ & $\left.\begin{array}{l}5.0 \times 10^{-4} \\
2.0 \times 10^{-4}\end{array}\right\}$ & 0.5 \\
\hline $\begin{array}{r}2 a \\
b\end{array}$ & $\begin{array}{l}49 \\
69\end{array}$ & $\begin{array}{l}30 \\
50\end{array}$ & - & 1.5 & $\begin{array}{l}1.7 \times 10^{-1} \\
6.6 \times 10^{-2}\end{array}$ & $\left.\begin{array}{l}4.3 \times 10^{-2} \\
1.8 \times 10^{-2}\end{array}\right\}$ & 1.5 \\
\hline $\begin{array}{r}3 a \\
b\end{array}$ & $\begin{array}{l}49 \\
69\end{array}$ & $\begin{array}{l}30 \\
50\end{array}$ & $\begin{array}{c}0.02 \\
-\end{array}$ & $\underline{0.5}$ & $\begin{array}{l}1.8 \times 10^{-5} \\
8.0 \times 10^{-5}\end{array}$ & $\left.\begin{array}{l}4.8 \times 10^{-5} \\
2.0 \times 10^{-5}\end{array}\right\}$ & 0.5 \\
\hline $\begin{array}{r}4 a \\
b\end{array}$ & $\begin{array}{l}49 \\
69\end{array}$ & $\begin{array}{l}30 \\
50\end{array}$ & - & 1.5 & $\begin{array}{l}1.4 \times 10^{-2} \\
6.4 \times 10^{-3}\end{array}$ & $\left.\begin{array}{l}4.4 \times 10^{-3} \\
1.8 \times 10^{-3}\end{array}\right\}$ & 1.5 \\
\hline $\begin{array}{r}5 a \\
b\end{array}$ & $\begin{array}{l}49 \\
69\end{array}$ & $\begin{array}{l}30 \\
50\end{array}$ & 0.002 & 0.5 & $\begin{array}{l}1.9 \times 10^{-5} \\
8.0 \times 10^{-6}\end{array}$ & $\left.\begin{array}{l}4.6 \times 10^{-6} \\
2.0 \times 10^{-6}\end{array}\right\}$ & 0.5 \\
\hline $\begin{array}{r}6 a \\
b\end{array}$ & $\begin{array}{l}49 \\
69\end{array}$ & $\begin{array}{l}30 \\
50\end{array}$ & - & 1.5 & $\begin{array}{l}1.3 \times 10^{-3} \\
6.4 \times 10^{-4}\end{array}$ & $\left.\begin{array}{l}5.0 \times 10^{-4} \\
1.8 \times 10^{-4}\end{array}\right\}$ & 1.5 \\
\hline
\end{tabular}

TABLE 1. Test of numerical procedure for determining curvature. $R_{a}=$ disturbance amplitude; $\omega=$ disturbance frequency; $N=$ number of collocation node points ; error = (exact - numerical) $/$ exact.

routine applies to the most severe geometry considered in this study and demonstrates the ability of the numerical scheme to resolve those details of the highly distorted shapes that will be important to this study. Larger amplitude disturbances or frequencies larger than $\omega \approx 2$ on this model shape would require more points to be resolved properly.

Finally, we should add that all calculations reported in this paper are done in double precision, integrals are evaluated using a five-point Gauss quadrature scheme and, in the vicinity of the singularity where rapid variation of the integrand occurs, special care is taken by subdividing the interval of interest into three smaller intervals prior to integrating. A small region, typically $10^{-3}$ to $10^{-4}$ the size of an interval, is cut out around the singularity and the integration over this portion is performed analytically (the singularity is integrable in the sense of a Cauchy principal value).

The interface shape is updated by solving the kinematic condition using an explicit Euler method. Preliminary calculations using a second-order Runge-Kutta method to update the shape gave nearly identical results. For the majority of shapes and eapillary numbers to be studied, the largest dimensionless velocities are, $O\left(\begin{array}{ll}0.1 & 1.0\end{array}\right)$ so the time-step chosen is typically $0.02-0.05+$ This value is reduced when regions of high curvature (and hence large local velocities) develop.

Our numerical procedure is then as follows. For a given shape solve for $\hat{\boldsymbol{u}}\left(\boldsymbol{x}_{s}\right)$, update the shape using the kinematic condition and repeat the procedure. Every few time-steps the collocation points are evenly redistributed based upon arclength along the interface. When a region of high curvature (the region of a neck during the end-

+ For larger viscosity ratios it is more convenient in the numerical calculations to scale the velocity with $u_{\mathrm{c}}=\sigma / \mu(1+\lambda)$ and the time with $t_{\mathrm{c}}=l_{\mathrm{c}} / u_{\mathrm{c}}$. In this way $\boldsymbol{u}=O(1)$ for all $\lambda$. For simplicity when comparing simulations involving different $\lambda$ we discuss all calculations using the timescale and velocity scale introduced in the text. 
pinching process - see figure 5) begins to develop, additional points are added in the immediate vicinity of the maximum curvature. Typically 5-8 additional points are added, at least tripling the local density of collocation points, which, coupled with the cubic spline used to represent the interface shape, maintains accurate resolution of the shape.

As we shall see, for these free-boundary problems the interface evolution can be quite complicated and knowledge of the internal and external velocity and pressure fields can be useful in understanding the observed behaviour. As mentioned following equation (2), this requires determination of both the interfacial velocity and interfacial stress. Hence, when a complete picture of the velocity and pressure fields is desired, the interfacial velocity is calculated as described above and a similar discretization process is used to solve for the unknown (interior) interfacial stress distribution, $\boldsymbol{n} \cdot \hat{\boldsymbol{r}}$. Once again, the unknown interfacial stress components are assumed to vary linearly over each element and the integral equation is recast as a system of linear equations which is solved using Gaussian elimination. Then, the exterior stress distribution, $\boldsymbol{n} \cdot \boldsymbol{T}$, is calculated using the stress boundary condition. Finally, the interfacial stress and velocity can be used in (1) and (2) to compute the velocity field at any interior or exterior location. A similar procedure can be followed to determine the interior and exterior pressure distribution in the fluid.

As a check on the numerics, a detailed comparison was made with the smalldeformation analysis of Barthes-Biesel \& Acrivos (1973) for drop deformation in axisymmetric extensional flows (briefly summarized in the next section) and with the velocity and pressure fields around solid prolate ellipsoids in extensional flows. In all cases, the comparison was excellent. Also, the drop volume was monitored as a function of time and typically changes by no more than a few tenths of a percent over several hundred iterations. For the drop relaxation studies to be described in $\$ 3.2$, a typical simulation required $600-3000$ iterations, hence resulting in accumulative volume changes from 1-10\% of the original drop volume. However, for simulations with $\lambda<O(0.01)$, the volume changes were larger (as other researchers have found). In these cases, the drop dimensions were rescaled every few time-steps in order to conserve volume. Comparing results with and without rescaling indicated that the qualitative behaviour (basically the overall shape and the critical conditions for drop burst) was unchanged when the rescaling was implemented, and the only significant change was a small change in the timescale for deformation.

\section{Results}

\subsection{Comparison of numerics with small-deformation theory}

We begin by briefly examining the deformation of an initially spherical droplet placed in an axisymmetric extensional flow $\boldsymbol{u}_{\infty}=\Gamma \cdot \boldsymbol{x}$, where

$$
\Gamma=\frac{\mathbb{C}}{2}\left(\begin{array}{rrr}
-1 & 0 & 0 \\
0 & -1 & 0 \\
0 & 0 & 2
\end{array}\right)
$$

and where the capillary number $\mathbb{C}=G a \mu / \sigma$. The appearance of $\mathbb{C}$ in (4) occurs because of our choice of the characteristic velocity as $u_{\mathrm{c}}=\sigma / \mu$ and the characteristic lengthscale as the undeformed drop radius, $a$. This numerical problem was considered by Rallison \& Acrivos (1978) and, as reported by Rallison (1984), higher accuracy results have been obtained by Duffy and Blundell for the special case 


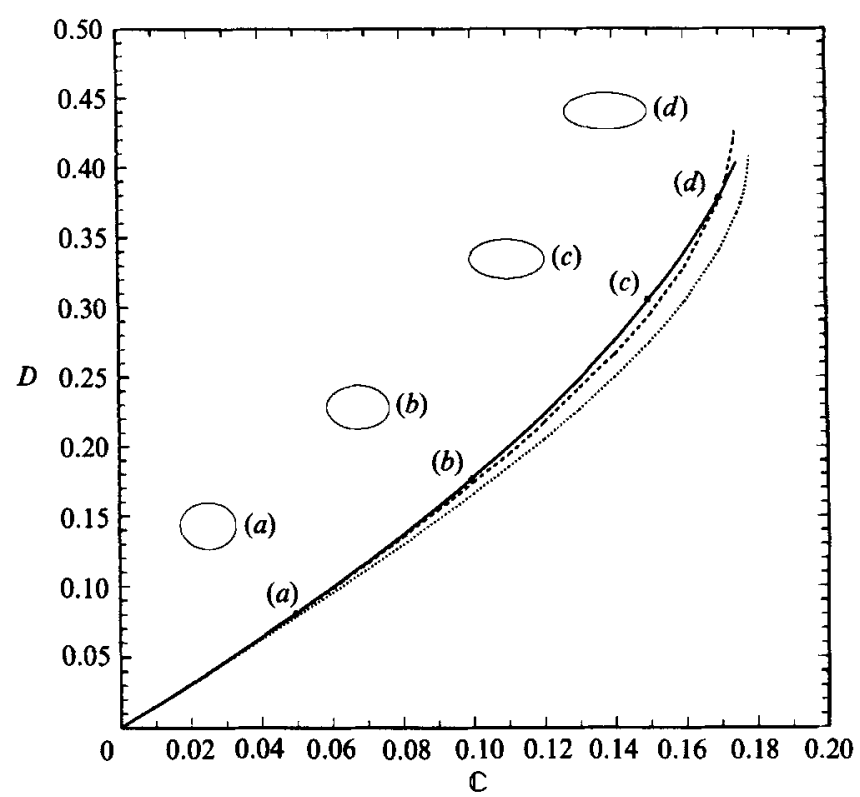

Figure 2. Drop deformation in a steady axisymmetric extensional flow : $D$ vs. $\mathbb{C} ; \lambda=0.1$. The solid line represents the numerical simulation and the dashed lines are the predictions of the smalldeformation theory of Barthes-Biesel \& Acrivos (1973). The short-dashed line denotes the $O(\mathbb{C})$ result and the long-dashed line the $O\left(\mathbb{C}^{2}\right)$ result. Several numerically generated steady shapes are included.

$\lambda=1$. The purpose of this section, as indicated earlier, is to indicate the accuracy of our numerical simulations by comparison with the small-deformation theory of Barthes-Biesel \& Acrivos (1973) and the numerical work mentioned above.

Theoretical work to predict the steady drop shape as a function of capillary number began with Taylor (1932). Taylor treated the drop as spherical, solved for the internal and external flow fields using continuity of velocity and tangential stress boundary conditions and determined the $O(\mathbb{C})$ correction to the drop shape by approximately satisfying the normal-stress balance at the fluid-fluid interface. Barthes-Biesel \& Acrivos (1973) extended the analysis to second order in capillary number and the applicability of this work and previous work was clarified by Rallison (1981). An additional discussion, including the extension to a large number of two-dimensional linear flows, can be found in Bentley \& Leal (1986).

For droplets that are only slightly deformed, an appropriate scalar measure of deformation is $D=(L-B) /(L+B)$, where $L$ and $B$ are the half-length and halfbreadth of the cross-sectional shape respectively. The deformation as measured by $D$ monotionically increases with increasing capillary number and, near the critical eapillary number, $D$ increases rapidly with small changes in $\mathbb{C}$.

A comparison between the present numerical results and existing theoretical results for $D$ versus capillary number is shown in figures $2-4$ for $\lambda=0.1,1.0$ and 10.0 respectively. The short-dashed and long-dashed lines denote the $O(\mathbb{C})$ and $O\left(\mathbb{C}^{2}\right)$ results from Barthes-Biesel \& Acrivos. Each of these analytic lines end when a capillary number is reached above which the theory predicts no steady shape. The numerical results are given by the solid line and, for completeness, several numerically generated shapes are shown in each figure. In figure 3, the open circles represent the computations of Duffy and Blundell (as reported by Rallison 1984) 


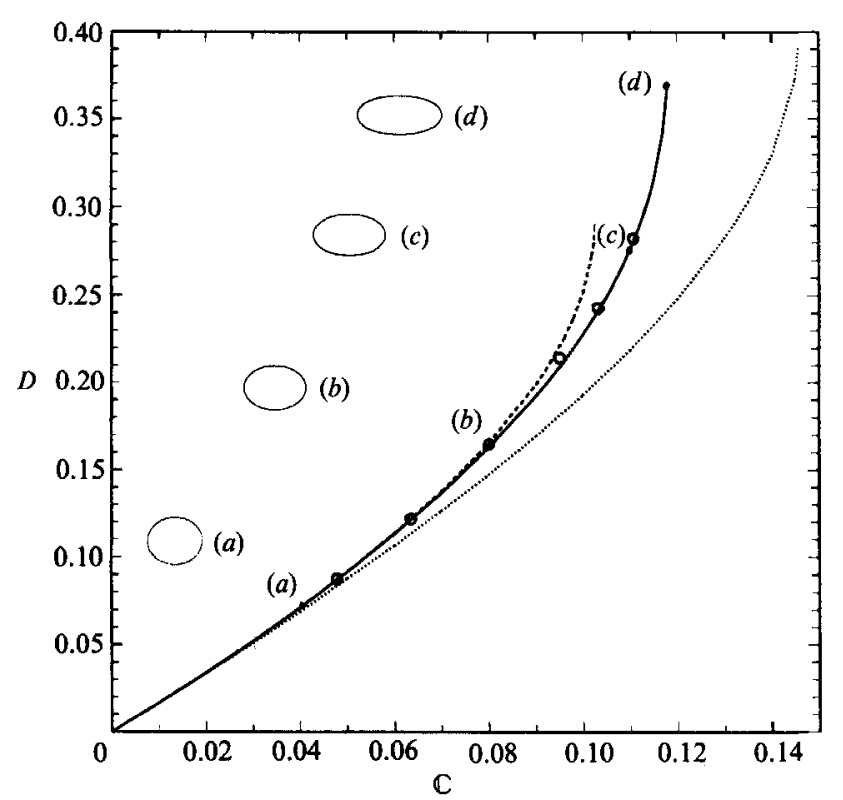

Figure 3. As for figure 2, but with $\lambda=1.0 ; \bigcirc$, calculations of Duffy and Blundell (as reported by Rallison 1984).

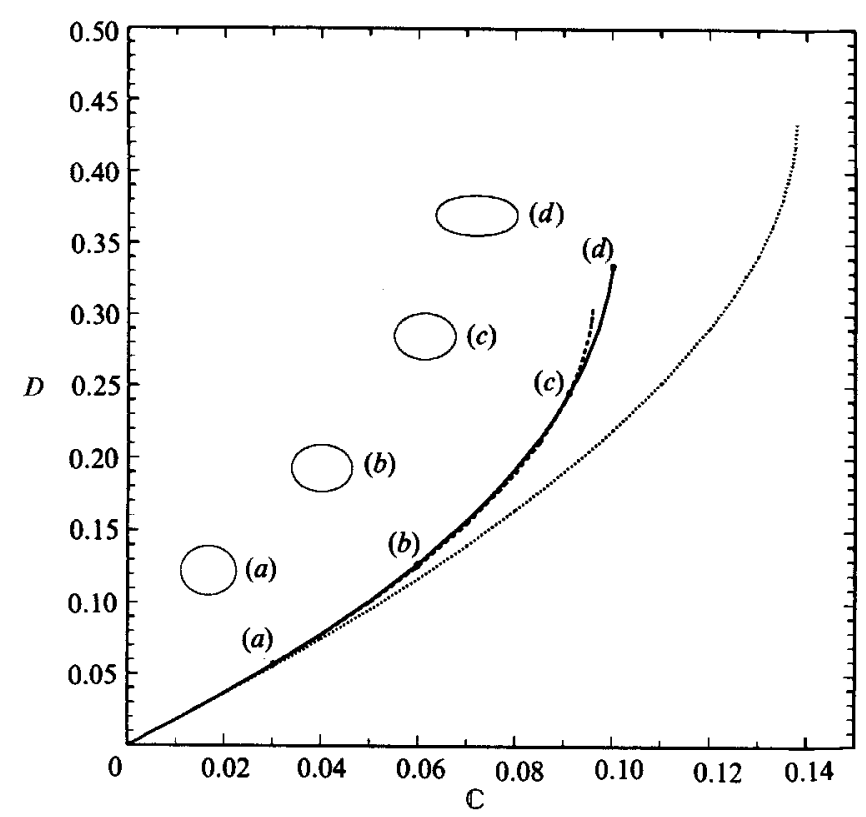

FIGURE 4. As for figure 2, but with $\lambda=10.0$.

which are clearly in excellent agreement with our numerics. The good agreement between analytic small-deformation theory and numerics is similar to the agreement observed by previous researchers upon comparison of the asymptotic theory with experimental results. The small-deformation theory does a remarkable job of capturing the major features of steady drop deformation even when the deformation is no longer small. 
With the level of accuracy indicated by this comparison and the numerical tests outlined in $\S 2$, we shall use the numerical method to examine the detailed dynamies of very highly extended droplets.

\subsection{Relaxation of extended droplets: the effects of viscosity ratio and initial shape}

We next demonstrate numerically some dynamical features associated with the relaxation and breakup of an initially extended drop in an otherwise quiescent fluid. Here we are not specifically concerned with how the droplet reached this highly deformed initial state. Rather, given a highly stretched droplet, the numerics describe the evolution of the drop shape. Although these results reiterate some aspects of the experimental study we reported previously (Stone et al. 1986), they illustrate the underlying mechanism of the end-pinching phenomenon, and contribute to a better understanding of drop breakup.

Our goals are twofold. First we wish to demonstrate that the numerics capture the qualitative features of the drop relaxation dynamics. This includes demonstrating that the low-viscosity-ratio experimental results previously reported are in accord with the same basic mechanism that is responsible for breakup at larger viscosity ratios, even though, at first, they may appear qualitatively different owing to the regions of very high curvature that are charactistic of nearly pointed, low-viscosityratio drops. Second, we seek to show that the computed results based upon the assumption of constant interfacial tension are in quantitative agreement with the experimental data from our earlier study, Stone et al. (1986).

It is convenient to characterize the degree of deformation using a single scalar parameter. For the highly elongated shapes that are typical of this part of the study, a dimensionless extension ratio, $L / a$, is appropriate, where $2 L$ is the end-to-end drop length.

With reference to (3), it is worth reiterating that in the absence of an imposed velocity gradient, i.e. $\mathbb{C}=0$, the only effect of the interfacial tension is to scale the velocity and, consequently, the time. Hence, for a given initial shape, the magnitude of the interfacial tension plays no qualitative role in the dynamical evolution of the drop shape, but only determines the timescale over which these dynamics occur. In particular, the qualitative features of the relaxation and breakup dynamics are completely determined by the viscosity ratio and the initial drop shape.

\subsection{1. $\lambda \geqslant O(0.05)$; shapes with bulbous ends}

In figure 5 we show a series of typical relaxation histories for $\lambda=0.05,0.1,1.0,7.5$, 10.0. The initial shape with $L / a=8.6$ is the same in each simulation and is taken from an experiment for $\lambda=11.3$. This highly stretched initial shape with bulbous ends is typical of experimental results for all $\lambda \geqslant 0.05$. The times reported have been made dimensionless with respect to the timescale $t_{\mathrm{c}}=R_{0} \mu / \sigma$ where the initial midsection radius $R_{0}$ has been chosen as the characteristic lengthscale. The timescale is independent of the viscosity ratio so that the different simulations may be compared directly as the viscosity ratio is changed. However, because the larger of the two fluid viscosities should be expected to provide the best measure of the temporal relaxation, we also list in parentheses the modified time, $t /(1+\lambda)$, corresponding to the characteristic timescale $i_{\mathbf{c}}^{*}=R_{0} \mu(1+\lambda) / \sigma$. This allows a test of the usefulness of this alternative measure of dimensionless time. These calculations provide a qualitative illustration of the effects to be expected when the viscosity ratio is varied.

The numerical scheme breaks down when end-pinching causes the local radius to 
$\left(\frac{t}{(1+\lambda)}=0,15.0,21.0,23.4\right)$

$t=0,15.75,22.05,24.57$

(a)

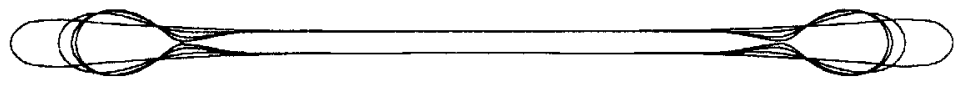

$(0,15.0,20.36,26.34,29.67)$

$0,16.50,22.40,28.97,32.64$

(b)

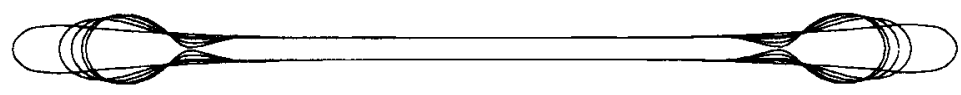

$(0,24.0,38.0,50.0)$

$0,48.0,76.0,100.0$

(c)

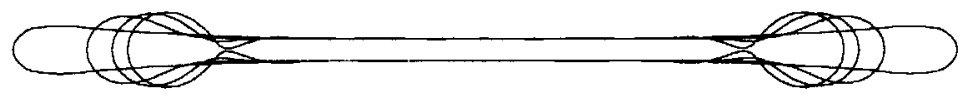

$(0,27.28,39.28,46.72,49.2)$

$0,231.9,333.9,397.1,418.2$

(d)

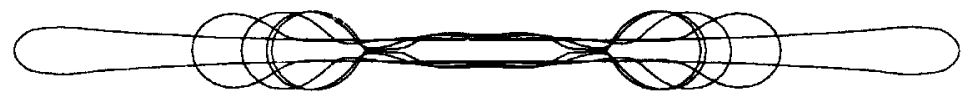

$(0,15.8,36.0,60.0)$

$0,173.9,396.0,660.0$

(e)

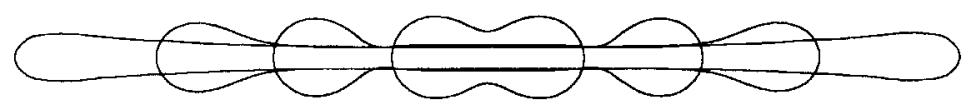

FigURE 5. Relaxation and breakup of an initially extended droplet in an otherwise quiescent fluid: dependence on viscosity ratio. The initial shape, $L / a=8.6$, is taken from an experiment for $\lambda=11.3$. This highly stretched initial shape with bulbous ends is typical of experimental results for all $\lambda>0.05$. (a) $\lambda=0.05 ;(b) 0.1 ;(c) 1.0 ;(d) 7.5 ;(e) 10.0$. The successive times $t$ are indicated on each figure together with the modified dimensionless times $t /(1+\lambda)$, shown in parentheses for reference.

become very narrow (typically $<0.003$ ) and the large local velocities calculated (corresponding to the large curvatures in this region) move collocation points across the droplet centreline. In a later simulation focusing on capillary-wave instabilities (\$3.3) we shall assume that actual fragmentation of the bulbous end from the central thread occurs at this point. Although this criterion is $a d$ hoc, if our primary purpose is to examine the evolution of the interface, then it nevertheless is a reasonable approximation, as the available experiments indicate very rapid breakup as the local radius thins. In this manner we may continue calculations beyond the initial fragmentation.

The results in figure 5 illustrate several interesting aspects of the relaxation physics. For small $\lambda$, the end of the droplet translates a relatively short distance prior to pinching. At the point where the ends appear close to breaking off, a central cylindrical thread remains which itself would undergo a similar end bulbing and 


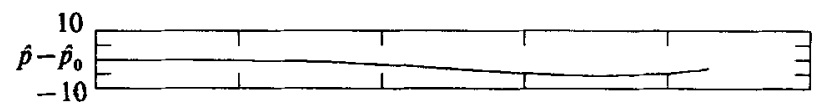

(a)
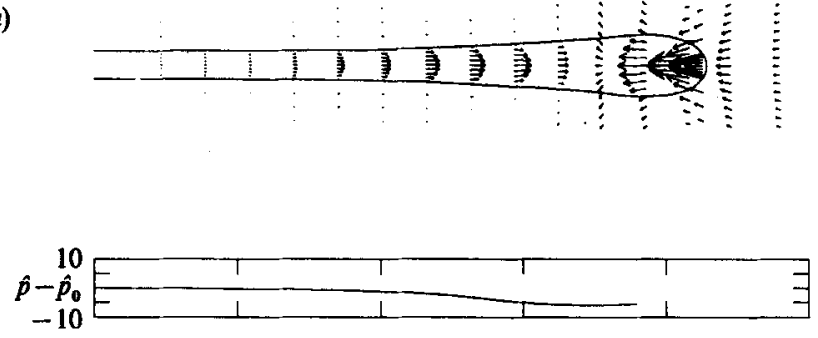

(b)
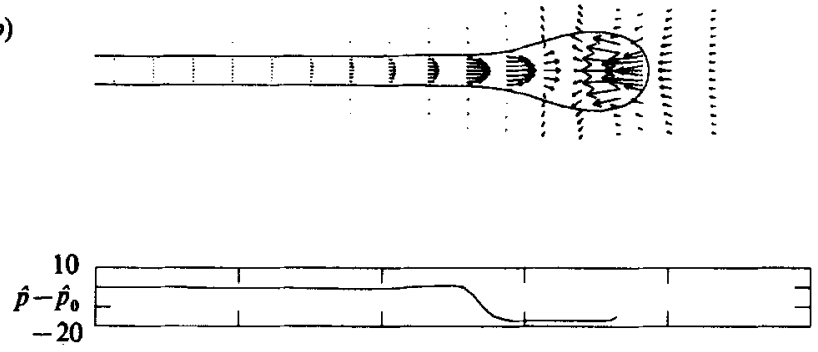

(c)

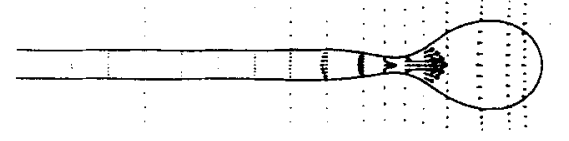

(d)

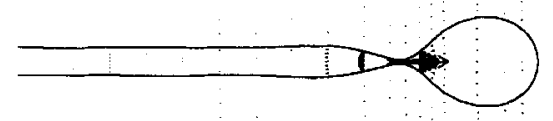

FraURE 6. Velocity and pressure fields for a relaxing drop in an otherwise quiescent fluid; $\lambda=0.1$. The pressure field $\hat{p}-\hat{p}_{0}$ shown is the droplet pressure along the centreline $(r=0)$ and is measured with respect to zero at the droplet centre $(r, z)=(0,0) .(a) t=0 ;(b) 22.40 ;(c) 28.97 ;(d) 32.64$.

relaxation process. It may also be noted that the rate of pinching accelerates as the pinch regions develop.

As the viscosity ratio is increased the relaxation process slows, as does the flow mechanism that leads to the development of a neck. Thus, the end of the drop moves closer to the midpoint prior to the development of a significant pinch. Indeed, for sufficiently viscous drops, no breakup occurs for the initial degree of extension considered in figure 5. In this case, the end of the drop translates all the way to the middle before a significant pinch can develop, and the drop simply returns to its equilibrium spherical shape. It may be added that the central section of the droplet remains cylindrical in all cases, and, at least for the elapsed time represented by these simulations, there is no evidence of (finite-amplitude) capillary waves. The simulations in figure 5 should be compared with figure 9 of our previous publication, Stone et al. (1986), which illustrates similar experimental observations.

From the dimensionless times $t /(1+\lambda)$ shown in figure 5 we observe that all the 


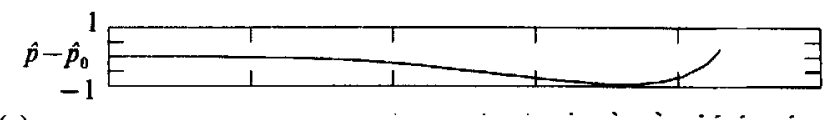

(a)
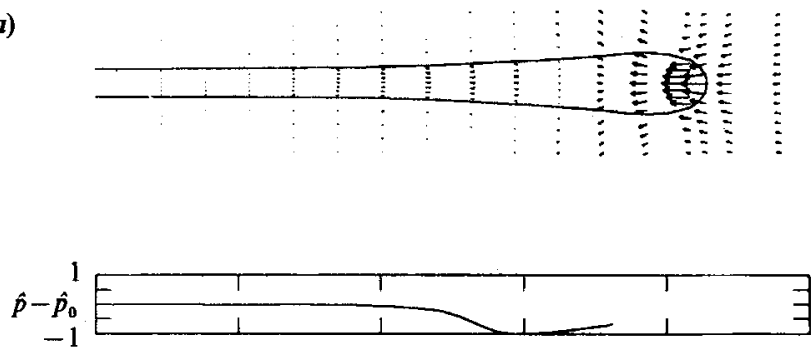

(b)
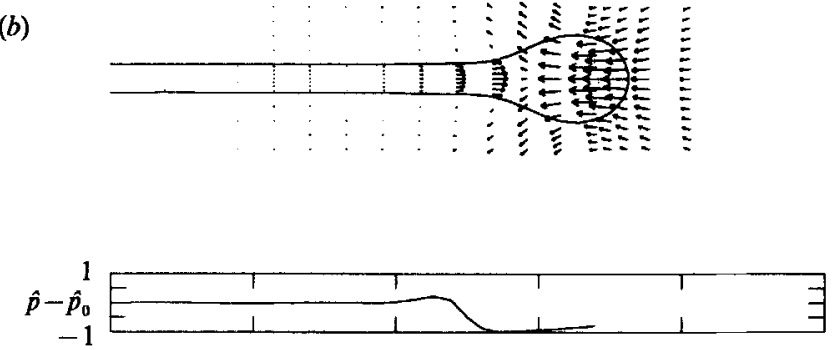

$(c)$
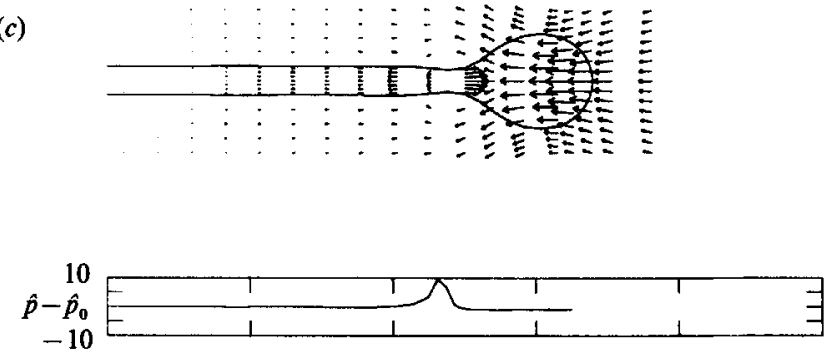

(d)

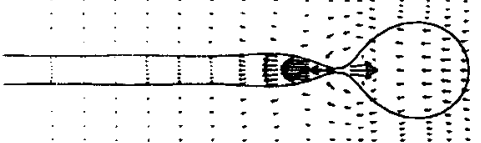

Figure 7. As figure 6 but with $\lambda=1.0 ;(a) t=0 ;(b) 48.0 ;(c) 76.0 ;(d) 100.0$.

relaxation and/or breakup processes occur for $25<t /(1+\lambda)<70$. Hence, this modified timescale yields a good measure of the dimensionless time over which significant changes in shape occur for a wide range of $\lambda$.

It is very informative to examine the interior and exterior velocity fields. These are shown in figures $6-8$ for $\lambda=0.1,1.0,10.0$, respectively, at different stages of the shape evolution. In each illustration the arrows denote the direction and relative magnitude of the fluid velocity. There is no connection between arrows in one simulation and those in another. Associated with each figure we show the variation of pressure in the droplet fluid, $\hat{p}$, along the droplet centreline. Since viscous forces are balanced by a pressure gradient, in each case we plot the difference $\hat{p}-\hat{p}_{0}$ where $\hat{p}_{0}$ is the pressure at the droplet centre $(z=0)$. When a significant pinch develops it is often difficult to resolve the interfacial stresses sufficiently to determine accurately the pressure variation in the pinch region and for this reason no pressure field is reported in figure $6(d)$.

From these developing velocity and pressure fields we make several observations. Qualitatively, we observe that for large viscosity ratios the interior velocity 


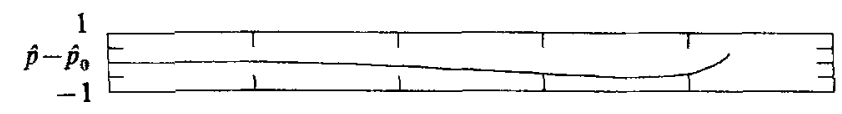

(a)
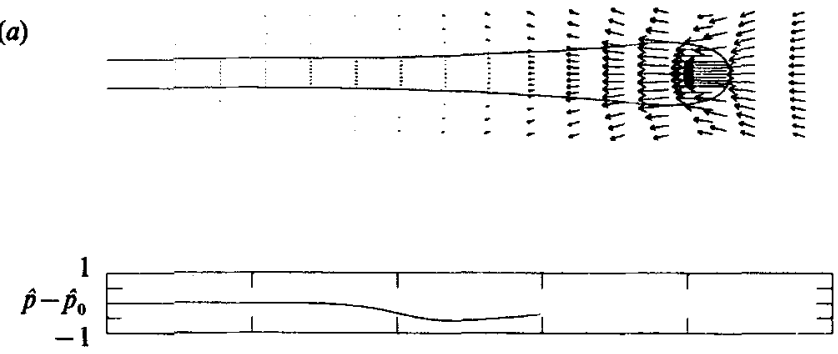

(b)
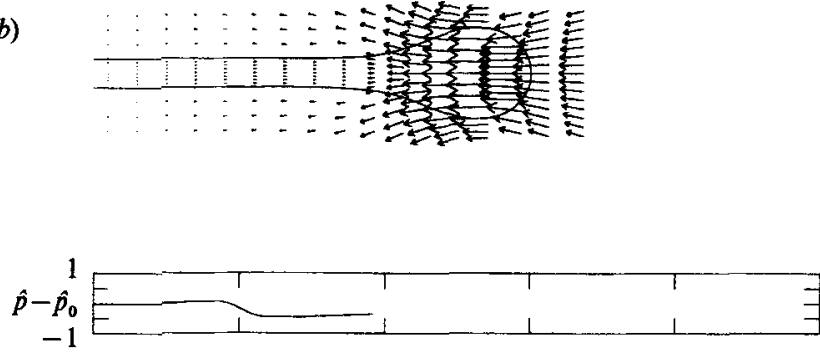

(c)
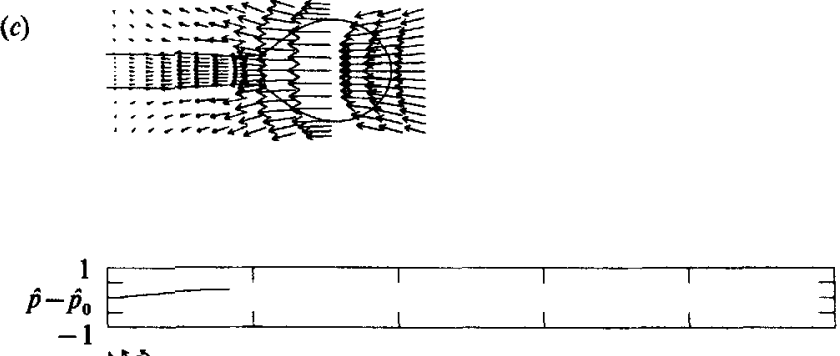

(d)

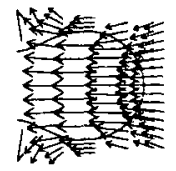

Figure 8. As figure 6 but with $\lambda=10.0$. (a) $t=0 ;(b) 173.9 ;(c) 396.0 ;(d) 660.0$.

gradients are small everywhere, while for small viseosity ratios large interior velocity gradients are possible. Also, there exist two relatively distinct regions of flow: the cylindrical region around the middle of the drop and the regions near the bulbous ends. For each viscosity ratio and for the initial shape shown, there is initially a pressure-driven flow in the cylindrical region toward the ends of the drop, and a counterflow from the bulbous ends toward the drop centre. This is true even for $\lambda=10$, where the velocities inside the cylindrical region are very small but are still directed towards the ends of the drop. In the case of $\lambda=1$, the 'collision' region where these flows meet is almost identical with the location of the pressure minimum. For larger $\lambda$, the relative magnitude of the flow from the ends is larger than that in the cylindrical region, and the zero velocity ('collision') point lies eloser to the droplet centre than does the pressure minimum. The converse is true for $\lambda<1$.

In all cases, the velocity profiles in the cylindrical region are parabolic as expected from a slender-body analysis (Acrivos \& Lo 1978). Also, we note that in the exterior fluid which is quiescent at infinity, the velocity field decays as $1 / \bar{\rho}$ (where 


$$
t=0,10.22,20.20
$$

(a)

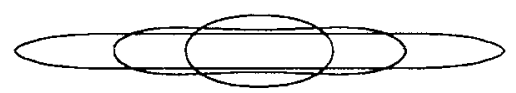

(b)

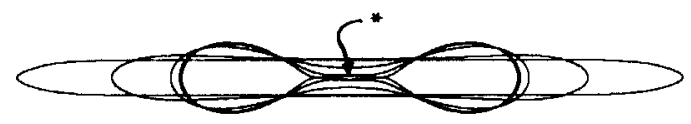

(c)

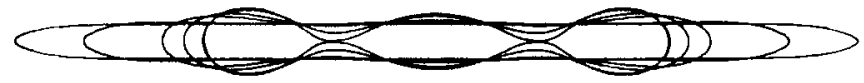

FigURE 9. Relaxation and breakup of low-viscosity-ratio droplets in an otherwise quiescent fluid; $\lambda=0.01$. The initial shape is taken from an experiment for $\lambda=0.011$. Low-viscosity-ratio droplets have long, slender shapes with nearly pointed ends. $(a) L / a=5.3 ;(b) 6.4 ;(c) 7.5$. Successive times $t$ are shown on the figure.

$\bar{\rho}^{2}=r^{2}+z^{2}$ ) sufficiently far from the droplet, characteristic of a Stokes flow (which, of course, is no surprise).

\subsection{2. $\lambda<O(0.01)$; shapes with nearly pointed ends}

In figure 9 we show a series of relaxation histories which are characteristic of the relaxation of relatively inviscid droplets. The viscosity ratio is 0.01 and the evolution of three successively elongated droplets $(L / a=5.3,6.4,7.5)$ is shown. The basic initial shapes are sketched from a series of experiments for $\lambda=0.011$. Notice that we have maintained the same ratio of midsection radius to radius of curvature at the end of the drop. These low-viscosity-ratio droplets have long slender shapes with nearly pointed ends. The high initial curvature at the ends of the drop results in large velocities near the ends and a very rapid initial reduction in drop length. However, if the drop is sufficiently extended so that this shortening process does not return it too close to its equilibrium spherical shape, the ends eventually bulb up which lowers the pressure in this region and the drop breaks by an end-pinching mechanism that is essentially the same as shown earlier for larger $\lambda$ values. Notice that even though the initial shape had a midsection that was very nearly cylindrical, capillary waves again play no significant role in determining the final drop size distribution as the end-pinching dynamics apparently occur on a timescale that is short compared with that required for growth of finite-amplitude capillary disturbances. We shall examine this in more detail in $\$ 3.3$.

In figure $9(b)$ it is interesting to notice that the thin cylindrical thread connecting 
(a)

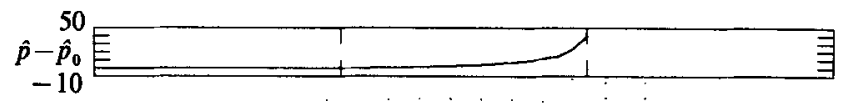

)
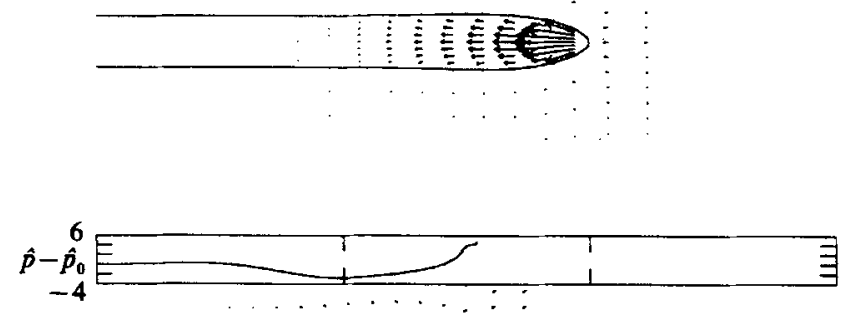

(b)
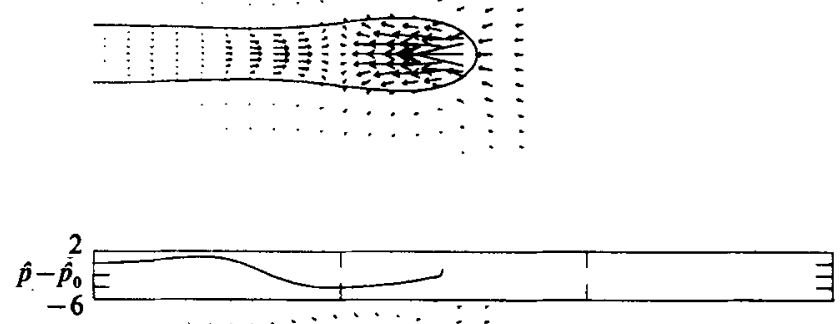

(c)

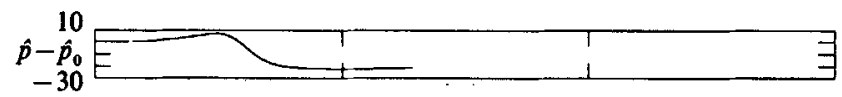

(d)

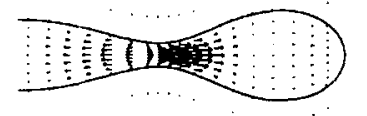

Figure 10. Velocity and pressure fields for a relaxing drop in an otherwise quiescent fluid; $\lambda=0.01$. The pressure field $\hat{p}-\hat{p}_{0}$ shown is the droplet pressure along the centreline $(r=0)$ and is measured with respect to zero at the droplet centre $(r, z)=(0,0)$. (a) $t=7.54 ;(b) 18.54 ;(c) 27.74$; (d) 29.74 .

the two bulbous ends at $t=22.68$ leads to the formation of a small satellite drop as the ends fragment a short time later. Because this is difficult to see we have indicated it by a star on the figure. This provides an example of satellite drop formation due to the nonlinear evolution of the drop shape. In figure $9(c)$, the more highly stretched initial shape leads to three almost equally sized daughter droplets.

In figure 10 the evolution of the velocity and pressure field is shown for the most highly stretched initial shape shown in figure 9 . The initial pressure gradient drives the rapid relaxation and as the end becomes more spherical this pressure gradient diminishes. It is clear that the pressure gradient in the central cylindrical region then begins to drive a flow toward the end of the droplet. This mass flux causes a neck to form in the cylindrical region, a pressure maximum develops and the breakup process accelerates.

Experimentally, the critical elongation necessary to fragment a drop with $\lambda=0.01$ lies in the range $5.4<L / a<6.2$. Hence, the numerics are in good agreement with 
this qualitative result of the end-pinching dynamics. Finally, by comparison of figures 5 and 9 , we can conclude that the different initial shape coupled with the lower viscosity ratio will lead to a noticeable change in the ultimate drop size distribution after breakup.

\subsubsection{The mechanism of end-pinching}

We are now in a position to provide a qualitative explanation of the relaxation and breakup of an extended liquid drop suspended in an otherwise quiescent fluid. As this reiterates several points made in our previous experimental study, we will be brief and stress those new aspects that have been uncovered by the numerical simulations.

In all cases we have examined, the initial shapes characteristic of the elongated droplets have basically cylindrical midsections. The pressure gradient in most of this region is virtually zero, hence very little flow occurs. However, a pressure gradient favouring flow toward the end does occur in the transition zone where the cylindrical midsection joins the bulbous end. One possibility is that this pressure gradient may lead to a flow that uniformly decreases the radius of the cylindrical midsection. However, the observed response is more localized. The pressure gradient generates a local flow from the cylindrical region toward the end which thus causes a neck to form in the drop shape. This is a precursor to end-pinching. Therefore, the mechanism for relaxation and breakup of an extended droplet in an otherwise quiescent fluid consists of a competition between a pressure-driven flow near the end, which causes translation of the end toward the droplet centre (thus tending to return the drop to its spherical equilibrium shape), and a pressure-driven flow away from the centre in the transition region which leads to the development of a neck and thus to breakup via a capillary pinch-off process.

Dynamics associated with $\lambda<1$ are characterized by relatively large internal velocity gradients and, for modest extensions, the net flux towards the centre of the droplet induced by end motion cannot inhibit formation of a neck and subsequent pinch-off. The pointed shapes typical of very low-viscosity droplets produce large velocities from the ends toward the droplet centre which, at least initially, inhibits the pinching process and allows a significant reduction in drop length prior to breakup (see figure 10). However, larger viscosity ratios are characterized by very small internal velocity gradients and the net flux induced by end motion, while initially retarded by the adverse pressure gradient and competing flux from the central section, can nevertheless dominate the flow in the transition region and thus inhibit breakup by inhibiting the formation of a pinch point. The consequences of this are that very large extensions of a viscous droplet (i.e. $\lambda \gg 1$ ) are necessary to guarantee breakup in an otherwise quiescent fluid. Since the effect of the formation of a pinch is to generate a local pressure maximum which retards motion from the end of the droplet, these large extensions are necessary to allow a significant pinch to develop prior to the end translating back to the droplet centre.

Overall, it is the global features, rather than any local details, of the initial drop shape that are responsible for the end-pinching dynamics. This observation was made in our previous experimental study (Stone et al. 1986). Similar remarks are made by Brady \& Acrivos (1982) in a study of inertial effects on the breakup of slender drops. It may be noted that a careful reading of the literature shows that the relaxation dynamics described above and in our previous experimental paper were first observed by Taylor (1934) and discussed by Grace (1971), though Grace incorrectly interpreted the breakup in terms of capillary-wave instabilities. 


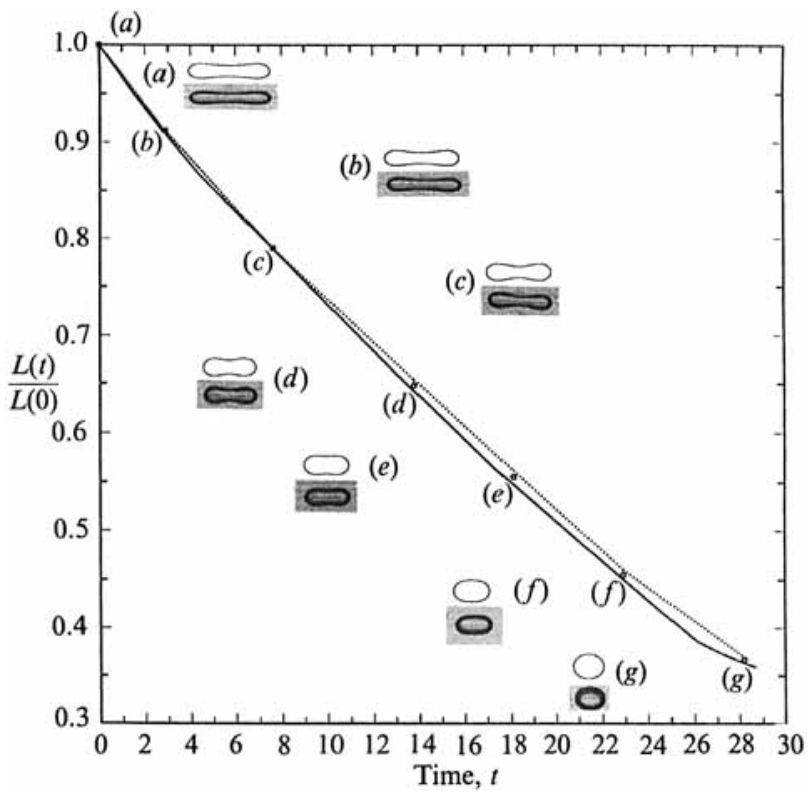

Figure 11. Quantitative comparison of numerical (solid curve) and experimental results (dotted curve) for relaxation of an initially extended droplet in a quiescent fluid; $\lambda=0.45-\mathrm{a}$ test of the constant-interfacial-tension boundary condition. Still photographs and corresponding numerically generated shapes are shown.

\subsubsection{Quantitative comparison of experimental and theoretical results}

Until this point, the numerical calculations have helped to explain the qualitative features of the end-pinching dynamics. One question which often arises in presentation of the experimental results is whether surfactant effects, producing interfacial-tension gradients, play any role in the observations. One way to address this question is to examine whether the present numerical results based on the assumption of constant interfacial tension can quantitatively predict the experimentally observed evolution of drop shapes.

Figures 11 and 12 compare experimental and numerical results for drop length as a function of relaxation time for two different viscosity ratios, $\lambda=0.45$ and $\lambda=11.3$, respectively. The experiments, conducted in a two-dimensional flow field generated in a four-roll mill, stretch the droplet at the critical capillary number and at some point during the elongation process the flow field is turned off (Stone et al. 1986). The Reynolds number $\sigma \rho R_{0} / \mu^{2}$ typical of the relaxation process is approximately $10^{-4}$ for these experiments. When the imposed flow is stopped abruptly the drop shape becomes axisymmetric on a timescale, $R_{0} \mu(1+\lambda) / \sigma \approx 0.1-1 \mathrm{~s}$, which is fast relative to the time of the overall relaxation process $(30-150 \mathrm{~s})$. Therefore, a direct comparison of the experimental results with the numerical calculations is valid. In these figures the dotted lines are the experimental results and the solid lines are the results of the numerical simulations. We also compare several photographs taken during the relaxation experiment with corresponding numerically generated shapes. The small differences observed are probably due to uncertainty in the exact value of the interfacial tension. In any case, the agreement between numerics and experiment illustrated in these figures is very good, both qualitatively and quantitatively. Clearly the timescale of the relaxation dynamics that we have observed is completely 


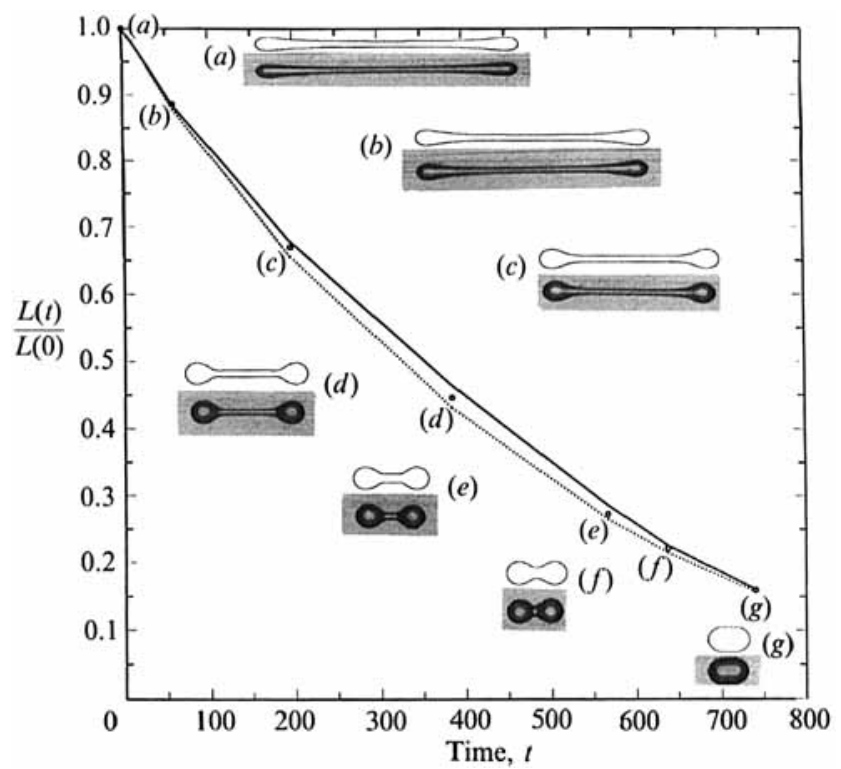

Figure 12. As figure 11 but $\lambda=11.3$.

captured using a constant value for the interfacial tension. A numerical study of drop deformation including the effect of variations in the interfacial tension, due to the presence of surfactants, is currently in progress.

\subsection{Capillary waves}

An important aspect of the breakup process which arises in an examination of the droplet relaxation dynamics, but has not yet been explicitly considered, is the role played by capillary waves. Existing investigations concerned with predicting drop size distributions have generally assumed that the drop could be approximated as an infinite fluid cylinder which fragmented owing to capillary-wave instabilities. Such theoretical studies, based upon linear stability theory, have been successfully compared with experimental studies of extremely long cylindrically shaped droplets by Rumscheidt \& Mason (1962) and Lee, Yu \& Flumerfelt (1981). However, as should be clear, the capillary instability mechanism cannot explain the droplet relaxation and breakup dynamics illustrated in the previous sections. Nevertheless, it is to be expected that if the droplet is very highly stretched prior to stopping the flow abruptly, then, while fragmentation still occurs at the ends owing to the endpinching mechanism, capillary waves should have enough time to evolve so as to play a role in the breakup of the central cylindrical portion of the droplet. Indeed, this premise was demonstrated experimentally by Stone et al. (1986).

In spite of some success in predicting the breakup of fluid cylinders, linear capillary-wave instability theory is not able to explain the existence or formation of satellite drops, nor is it applicable in a self-consistent sense when the disturbances reach a finite amplitude as must inevitably occur in breakup. The goal of this section is to use the boundary-integral method to obtain a more complete understanding of drop breakup in the presence of (finite-amplitude) capillary waves. Since the later stages of the breakup process involve finite-amplitude capillary waves growing on a cylindrical fluid thread, the numerics also shed light on this simpler, though classic, problem. 


\begin{tabular}{|c|c|c|c|c|c|}
\hline \multirow{3}{*}{$\begin{array}{c}\lambda \\
\text { viscosity } \\
\text { ratio }\end{array}$} & & \multicolumn{4}{|c|}{$R_{a}=$ initial disturbance amplitude } \\
\hline & & $10^{-8}$ & $10^{-6}$ & $10^{-4}$ & $10^{-2}$ \\
\hline & $\begin{array}{c}\gamma \\
\text { growth }\end{array}$ & \multicolumn{4}{|c|}{ Time for growth } \\
\hline $10^{-3}$ & 0.399 & 44.4 & 32.9 & 21.3 & 9.8 \\
\hline $10^{-2}$ & 0.266 & 66.6 & 49.2 & 31.9 & 14.6 \\
\hline $10^{-1}$ & 0.109 & 163.2 & 120.8 & 78.4 & 36.0 \\
\hline 1 & 0.0355 & 499.4 & 369.6 & 240.0 & 110.2 \\
\hline 10 & $9.82 \times 10^{-3}$ & $1.8 \times 10^{3}$ & $1.3 \times 10^{3}$ & 871.2 & 400.4 \\
\hline 100 & $1.45 \times 10^{-3}$ & $1.2 \times 10^{4}$ & $9.1 \times 10^{3}$ & $5.9 \times 10$ & $2.7 \times 10$ \\
\hline
\end{tabular}

TABLE 2. Time for capillary wave to grow to half the cylinder radius as a function of the disturbance frequency and amplitude for different viscosity ratios. The maximum growth rate is also listed.

A few remarks regarding capillary waves on cylindrical fluid interfaces are in order. First, it may be noted that an infinite, stationary fluid cylinder suspended in an otherwise quiescent fluid does represent an equilibrium solution to the governing equations and boundary conditions (unlike an elongated drop of finite length). This stationary solution, however, is unstable to infinitesimal disturbances with wavelength greater than the cylinder circumference. Linear stability theory (e.g. Lee \& Flumerfelt 1981) examines the evolution of an arbitrary disturbance by superposition of individual Fourier modes; i.e. the fundamental disturbance has the form $\mathrm{e}^{\gamma t} \cos (\omega z)$ and an eigenvalue problem is formulated for the growth rate $\gamma(\omega)$ as a function of the disturbance frequency $\omega$. The disturbance growth rate also depends on the viscosity ratio.

The linear theory implicitly assumes that all disturbances are equally likely and that the observed drop sizes on breakup will correspond to the wavelength of the fastest growing linear mode. If the initial amplitude of this critical disturbance is known, then the time for breakup can be estimated. For example, table 2 shows, for different viscosity ratios, the time (made dimensionless with respect to $t_{\mathrm{c}}=R_{0} \mu / \sigma$ ) necessary for a capillary wave to grow to half the cylinder radius as a function of the initial disturbance amplitude. The most important point is that an initial disturbance with amplitude $10^{-4}$ takes longer to evolve to half the cylinder radius than the time necessary for the examples of end-pinching that are shown in figure 5. This helps to explain why the majority of our experimental and numerical studies do not exhibit capillary waves; the end-pinching dynamics (at least the first fragmentation of the end) evolve on a faster timescale than is necessary for the growth of an initially infinitesimal disturbance to finite amplitude.

In order to understand more completely the development of capillary waves, including any nonlinearities related to finite-amplitude disturbances, we numerically examine the evolution of an initial disturbance on the surface of a highly stretched droplet with a uniform cylindrical midsection. An initial disturbance is generated on the cylindrical region by the superposition of sinusoidal disturbances of several different frequencies (including modes that, according to linear theory, are both stable and unstable) and the growth of the disturbance from small to finite amplitude is observed. As a means of characterizing the details of the interface evolution beginning with an arbitrary disturbance, a Fourier decomposition of the drop shape 
is performed. Since the drop shape is fore-aft symmetric, the amplitude of a given frequency is calculated from

$$
\hat{F}(\omega)=\int_{0}^{l}\left[r(z)-r_{\text {avg }}\right] \cos (\omega z) \mathrm{d} z,
$$

where $r(z)$ represents the interface position, $r_{\text {avg }}$ represents the average interface position, and $l$ denotes the axial position where the integration is terminated. $r_{\text {avg }}$ changes slowly as a function of time owing to the flow accompanying the droplet relaxation and the growth of capillary waves. Truncating the integration at $l$ is necessary because the droplet is not infinite and we wish to isolate capillary wave dynamies from end-pinching dynamics. Although the choice of $l$ and the number of collocation points used to represent the interface make a small difference in the exact values of the Fourier amplitudes calculated from (5), the qualitative trends we observe are not changed. The resolution of the Fourier decomposition is affected in two ways: (i) because the discretization of the initial shape has only about one point every 1.5 units in the $z$-direction, we are not able initially to resolve disturbances for $\omega>2$; and (ii) as the integration in (5) is truncated at $z=l$, the numerical Fourier transform cannot accurately resolve details for frequencies $<\pi / l$.

In figure 13 we illustrate the complete evolution of a highly extended drop, $\lambda=1$, in an otherwise quiescent fluid. Numerical calculations using $\lambda=1$ are convenient since they produce a very simple form of (3) but still highlight the important dynamics. The drop has a dimensionless initial extension ratio $L / a=14$ and the drop half-length is initially 73 times the radius of the cylindrical region. Although the shape is rather extreme, we shall see that even with only 49 collocation points the numerics can resolve the basic qualitative and quantitative features of capillary wave growth. The cylindrical midsection has a small disturbance superposed on it with an initial amplitude about $3 \times 10^{-3}$. This amplitude is large enough that numerical errors do not play a significant role in the early development of the capillary wave, yet small enough that the results should approximate those from linear theory. The disturbance is made up of a number of discrete modes and the Fourier decomposition of this disturbance is shown in figure $14(a)$.

The droplet in figure 13 fragments initially at the ends owing to the end-pinching process and fractures near the centre at later times owing to the growth of finiteamplitude capillary waves. For numerical ease we have made the approximation that when the numerics indicate fracturing is complete (numerical difficulty occurs when large local velocities in the region of a pinch move collocation points across the droplet centreline as discussed in $\$ 3.2 .1$; e.g. figure $13 d, k, m$ shows the droplet shortly before fragmentation occurs) the end droplets are assumed to be broken off (the radius at the point of fracture is set equal to zero), the calculation is started again with the central thread only and the daughter droplets are neglected completely in ensuing calculations. Because the fracturing process creates very high curvatures at the ends of the central thread which lead to a very rapid relaxation and bulbing of the ends, the approximation made by neglecting the fragmented daughter droplets should have little effect as the calculations are continued. In other words, even though the pinch-off criterion is ad hoc, it plays no role in our results as the timescale associated with the bulbing process at the nearly pointed ends of the remaining central thread is very fast compared with the timescale of the capillarywave growth process. In figure 14 we plot the square of the amplitude of the Fourier decomposition as a function of frequency, calculated using (5) with $l=20$, at intermediate times during the relaxation process. Practically identical results were 
$(n)$

(m)

(e)

(d)
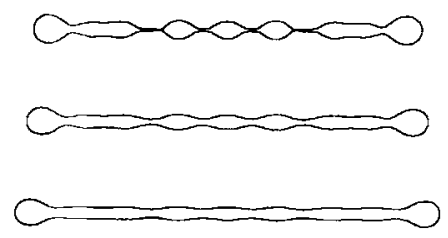

(h)

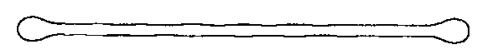

$(g)$

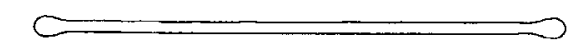

()

(c)

(b)

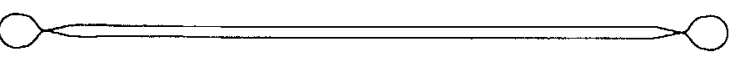

)

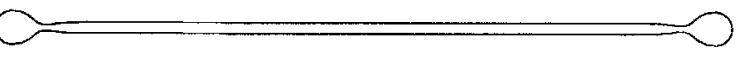

)

(a)

FIGURE 13. Evolution of capillary waves during the relaxation/breakup of an initially highly extended droplet suspended in an otherwise quiescent fluid. Initial shape: $L / a=14, \lambda=1.0 .(a)$ $t=0 ;(b) 30.0 ;(c) 50.0 ;(d) 63.4 ;(e) 64.6 ;(f) 73.0 ;(g) 104.6 ;(h) 170.6 ;(i) 194.6 ;(j) 218.6 ;(k) 234.6$; (l) $235.2 ;(m) 240.8 ;(n) 242.0$. When fragmentation occurs, the daughter droplets shed from the end are completely neglected as the calculation is continued. Notice the formation of small satellite droplets in the latter stages of the capillary breakup of the cylindrical midsection.

found with $l=25$. Notice that the vertical scale in the plots changes as time increases.

We make the following observations concerning the relaxation and breakup process. Initially, all the interesting behaviour occurs in the vicinity of the end of the droplet where the curvature variations are the largest, figure $13(a-d)$. End pinching causes a droplet to be shed at about $t=64$. Then, the very high curvature at the end of the remaining central thread causes rapid bulbing of the end and a significant relaxation. Capillary waves are visible (finite amplitude) at $t=100$ and are very evident at $t=200$. At $t=234.6$ the end of the droplet appears ready to fragment owing to a combination of the end-pinching mechanism and the capillary instability mechanism, leaving a central thread consisting of three droplets connected by thin cylindrical threads. The final fragmentation, figure $13(l-n)$, produces three equally sized main drops and two smaller satellite droplets. First, the two outside drops in 
(i)

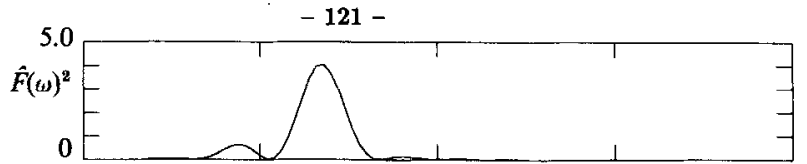

(h)

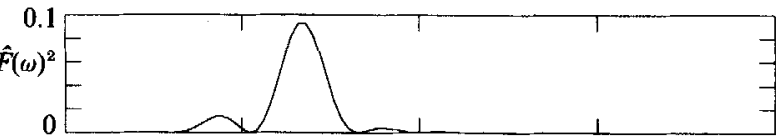

$(g)$

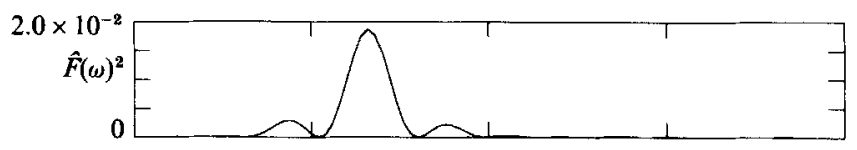

(f)

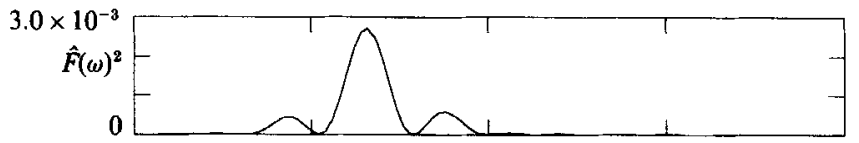

(e)

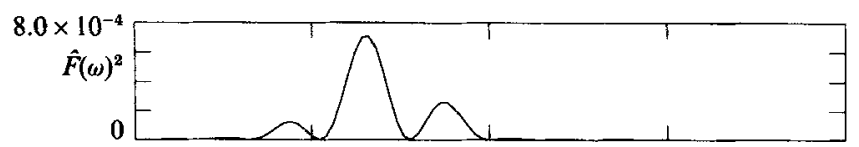

(d)

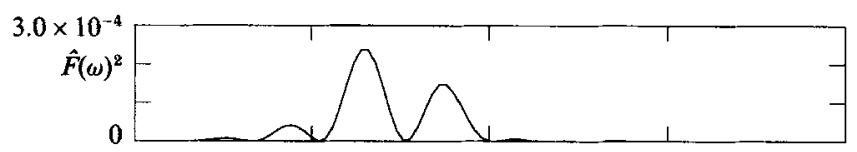

(c)

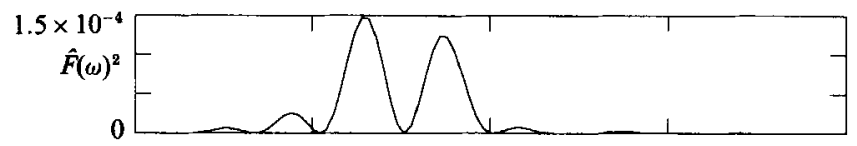

(b)

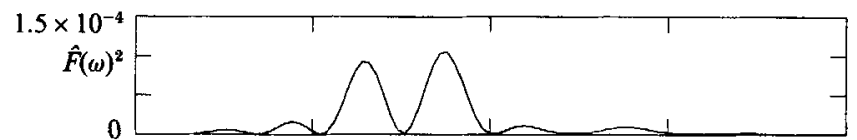

(a)

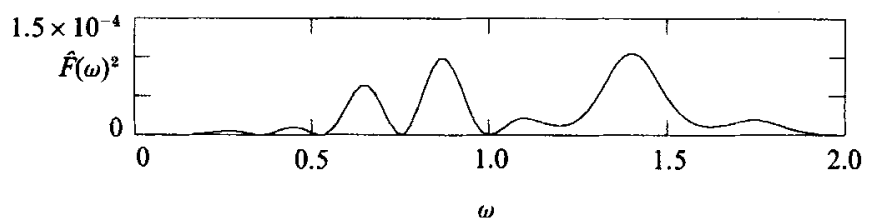

Figure 14. Evolution of the Fourier spectrum of the disturbance on the central cylindrical region of the drop. Fourier decomposition calculated using equation (5) with $l=20$. (a) $t=0 ;(b) 10.0 ;(c)$ $20.0 ;(d) 30.0 ;(e) 50.0 ;(f) 73.0 ;(g) 104.6 ;(h) 170.6 ;(i)$ 194.6. Modes that are stable in the linear stability theory are $\omega>1$. All $0<\omega<1$ are unstable according to the linear theory. Notice that the dominant mode initially is a linearly stable mode and it rapidly decays.

figure $13(l)$ fragment and the middle drop begins to pinch off from the thread-like regions. Continuation of the simulation shows formation of two satellite drops in figure $13(n)$. Clearly, the satellite drops form from the cylindrical threads that originally connect the larger drops. The formation of satellite drops from the cylindrical thread between two bulbous reservoirs occurs following the formation of pinch points at the ends of the cylindrical region. This process, involving a cylindrical region connecting bulbous 'ends', appears very similar to the basic end-pinching dynamics originally outlined in our previous study (Stone et al. 1986). The formation of satellite drops from the cylindrical connecting thread has been observed by, among others, Goren (1964) and Goedde \& Yuen (1970). It is clear that satellite drop 
formation is a direct consequence of the nonlinear dynamics of interface evolution. In a related study of the breakup of liquid filaments, Bousfield et al. (1986) do not observe satellite drop formation in the limit of negligible inertial effects. We have no explanation for this discrepancy other than the observation that their simulation only considers a viscous thread, neglecting the environment (limit $\lambda \rightarrow \infty$ ).

In addition, we should mention that this numerical simulation has several features in common with the experimental observations of Rutland \& Jameson (1971) concerning the formation of secondary undulations which form on the cylindrical thread connecting the primary disturbances growing on a liquid jet. These secondary undulations were explained by the nonlinear theory of Yuen (1968) in which inertial effects were assumed to dominate. It is very difficult to distinguish between our observations of the latter stages of drop breakup (figure 13l-n), which appears to be a deterministic consequence of nonlinear interface dynamics associated with a cylindrical thread connecting two bulbous reservoirs, and the instability mechanism observed in the above studies on jet breakup which is a result of growth of harmonics associated with the primary mode of instability.

It is interesting that the capillary-wave development near the central region of the droplet is so uniform in spite of the fact that the droplet is finite and continually shortening. This suggests that there is very little flow in this central region, and hence very little effect on the capillary-wave dynamics due to the continuous shortening of the drop due to the flow near the ends. The velocity profiles illustrated previously in $\$ 3.2$ support this idea. It is for this reason that the quantitative comparison with linear theory examined below is carried out for a thread in a quiescent fluid rather than a thread in an extensional flow (e.g. Mikami et al. 1975).

The Fourier decompositions shown in figure $\mathbf{1 4}$ are also very informative. Initially the dominant frequency is $\omega=1.4$, a linearly stable mode. This was purposely chosen in order to shed light on the evolution of stable modes in a case when their initial amplitudes were larger than that of any other (unstable) modes. Very rapidly this stable mode decays (as expected from linear theory) and the new dominant modes lie within the linearly unstable range $0<\omega<1$. These results demonstrate that the numerics can resolve the stabilizing influence of interfacial tension for the smallamplitude, short-wavelength disturbances. The very rapid decay of this linearly stable mode occurs at a rate comparable with that predicted by linear theory although the growth rate is difficult to calculate accurately as the amplitude quickly decays to a point where numerical error is significant. As the disturbance continues to grow on the cylindrical interface, three modes that are present initially dominate the Fourier spectrum, $\omega=0.44,0.66,0.88$. For $\lambda=1$, the most unstable mode predicted by linear theory is $\omega=0.56$. However, the rate of growth of the modes that we do see is in qualitative accord with expectations from linear theory. While each of these three dominant modes have positive growth rates, the frequencies $\omega=0.44$, 0.66 have growth rates, according to linear theory, approximately twice that of $\omega=0.88$ and, indeed, examination of the Fourier spectra in figure 14 demonstrates that these former two modes overtake the latter mode as time progresses.

A quantitative comparison of the rate of growth of the amplitude of the different frequencies with predictions of linear stability theory is illustrated in figure 15. This figure shows a plot of $\ln \hat{F}^{2}$ versus time for the three frequencies, $\omega=0.44,0.66,0.88$, in addition to the most unstable frequency (from linear theory), $\omega=0.56$. It is only the slope of the curves that matters as the slope is directly proportional to the disturbance growth rate. The plot is stopped at $t \approx 200$ when the disturbance has 


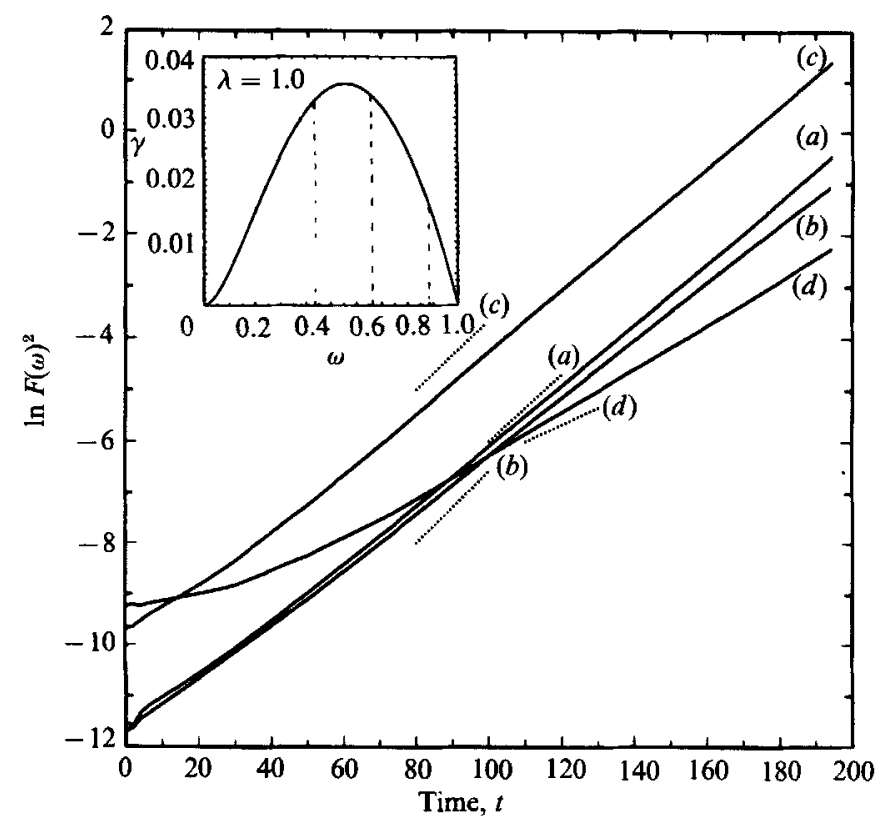

FIGURE 15. Comparison of numerical results with predictions of linear stability theory, $\ln \hat{F}(\omega)^{2} v s$ dimensionless time, $t$, for different frequencies. The solid lines are the numerical results and the dashed lines are the predicted slopes from linear stability theory. $(a) \omega=0.44 ;(b) 0.56 ;(c) 0.66 ;(d)$ 0.88 . The inset shows the stability diagram versus $\omega$ for $\lambda=1.0$. Notice that there exists a relatively wide range of frequencies with growth rate near the maximum value.

grown to a finite amplitude of about 0.2 and the droplet has shortened to a point where the choice of $l$ in the Fourier decomposition is no longer a simple matter. The solid lines are the numerical calculations and the dashed lines are the predicted slopes from linear stability theory. The results are quite good considering the numerical approximations and the very extreme initial shape, and indicate that the linear theory is good even as the disturbance evolves to finite amplitude. We have no explanation for the fact that the $\omega=0.88$ (curve $d$ ) data show more deviation from the linear theory at later times than the other frequencies, nor for the observation that it is not straight initially, unlike the other curves.

Perhaps most interesting is the observation that the dominant mode corresponds to $\omega=0.66$ and is responsible for the final drop size distribution on the central region of the droplet. The most unstable linear mode has a much smaller initial amplitude and is never able to catch up even through it has a larger growth rate. The fact that the most unstable linear mode, $\omega=\mathbf{0 . 5 6}$, does not dominate appears due to the very small differences in growth rate between this 'critical' frequency and nearby frequencies. This is indicated in the inset to figure 15 which shows a stability diagram with the growth rate $\gamma$ plotted as a function of the disturbance frequency $\omega$ for $\lambda=1$. In the neighbourhood of $\omega=0.56$ there is a wide range of frequencies with growth rates close to the maximum or 'critical' value. For these frequencies the evolution depends primarily on the magnitude of the initial disturbance.

At the later times when the choice of $l$ is difficult, we plot $\ln u_{r}(z=0)$ versus time. For a disturbance of a given frequency, linear stability theory predicts this graph to be linear with slope $\gamma$. This is illustrated in figure 16 . The solid line represents the numerical simulation and the dashed line is simply a straight line with the same initial slope. The growth rate calculated from this line is $\gamma=0.030$, which is in 


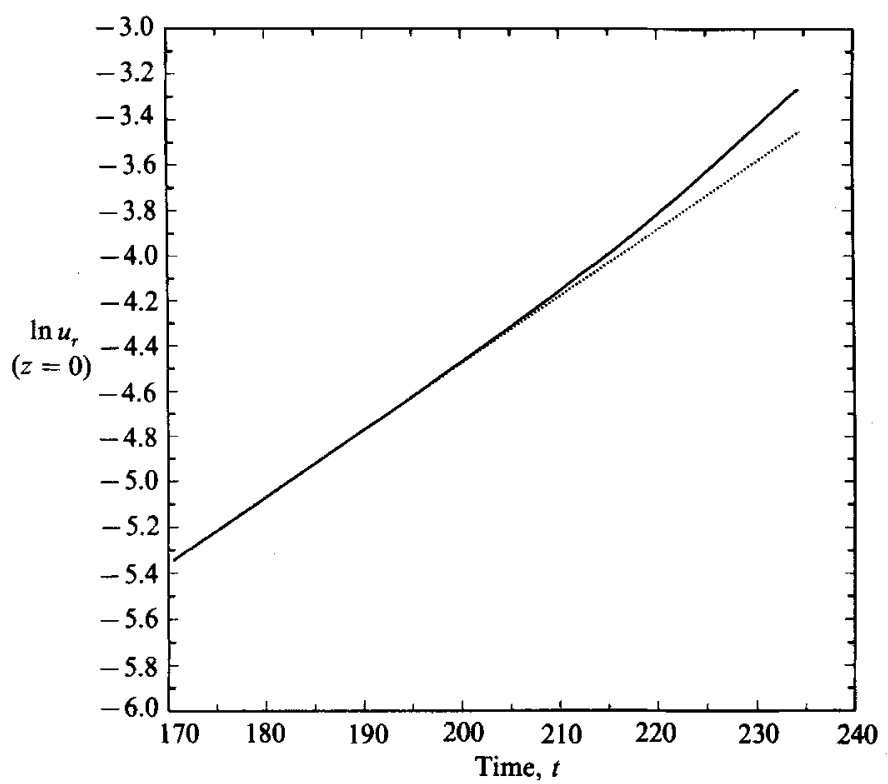

Figure 16. $\ln u_{r}(z=0)$ versus $t$. The solid line represents the numerical calculations. The dashed line is a straight line with the same initial slope. The slope of this line yields a growth rate of $\gamma=0.030$ which is in good agreement with the prediction of the linear theory based on the dominant mode, $\gamma(0.66)=0.033$. At later times the interface evolves more rapidly than the linear theory predicts.

reasonable agreement with the growth rate, predicted from linear theory, at the dominant frequency, $\gamma(0.66)=0.033$. Remarkably, the linear theory holds even though the disturbance is clearly finite amplitude. Very close to the point of actual fragmentation the interface evolves more rapidly than theory predicts, which demonstrates that nonlinear effects do eventually become noticeable, but only in the latter stages of the breakup process on the cylindrical thread-like region.

The simulation represented in figures 13-16 highlights several dynamical features associated with capillary wave growth on cylindrical fluid interfaces. Perhaps the most intriguing of the observations is the fact that the linear theory is remarkably good even when the disturbance is no longer small and even though the cylinder is finite and continually shortening. The nonlinearities associated with the evolution of capillary waves are only evident close to the point of actual fragmentation and their only significant consequence appears to be the formation of the small satellite drops in the final disintegrated drop. We also note that the numerics did not select the linearly most unstable disturbance. Instead, the linearly unstable mode with the largest amplitude at $t=0$ simply dominated for all times. This appears to be a consequence of the fact that the linear growth rates in the neighbourhood of the critical mode differ by only a few percent so that for significant differences in initial amplitude, the most unstable linear mode is never able to catch up. Of course, if the initial disturbances were truly infinitesimal, the results from linear theory would apply.

A simulation was also performed with the largest initial disturbance chosen to be coincident with the linearly most unstable mode, i.e. $\omega=0.56$. In this case the interface evolved as in figure 13 but the predominant disturbance remained $\omega=0.56$ for all times and the growth rate calculated numerically for $\omega=0.56$ differed from 
the prediction of linear stability theory by less than $6 \%$. As mentioned previously, the qualitative observations made during these numerical simulations of finiteamplitude capillary waves on stationary cylindrical interfaces are very similar to the observations made in related studies on the breakup of inviscid liquid jets (Goedde \& Yuen 1970).

Finally, we recall that in our complementary experimental study of capillary waves (Stone et al. 1986, figure 13), there was excellent agreement between the linear theory and experiment for $\lambda=0.1$ and 1.3, but poor agreement for $\lambda=12.2$. The difference for the higher viscosity ratio suggests that the flow from the end due to the relaxation process that is characteristic of very viscous drops may modify the selection of the most unstable wavelength. It may be noted, in this regard, that the growth rate versus frequency diagram is very flat for $\lambda>10$ so that there is a large range of frequencies with growth rates about equal to that of the most unstable linear mode. Hence, a small modification due to end effects may play an important role.

This work was supported by a grant from the fluid mechanics program of the National Science Foundation. One of the authors (H.A.S.) was partially supported through an IBM Graduate Research Fellowship.

\section{REFERENCES}

Acrivos, A. 1983 The breakup of small drops and bubbles in shear flows. Ann. NY Acad. Sci. 404, $1-11$.

Acrivos, A. \& Lo, T. S. 1978 Deformation and breakup of a slender drop in an extensional flow. J. Fluid Mech. 86, 641-672.

Ascoli, E. P. 1987 Low-Reynolds number hydrodynamic interaction of a solid particle with a planar wall. MS thesis, California Institute of Technology.

Barthes-Bieser, D. \& Acrivos, A. 1973 Deformation and burst of a liquid droplet freely suspended in a linear shear field. J. Fluid Mech. 61, 1-21.

Bentley, B. J. \& Leal, L. G. 1986 An experimental investigation of drop deformation and breakup in steady two-dimensional linear flows. J. Fluid Mech. 167, 241-283.

Bogy, D. B. 1979 Drop formation in a circular liquid jet. Ann. Rev. Fluid Mech. 11, 207-228.

Bousfield, D. W., Keunings, R., Marrucci, G. \& Denn, M. M. 1986 Nonlinear analysis of the surface tension driven breakup of viscoelastic flaments. J. Non-Newtonian Fluid Mech. 21, $79-97$.

Brady, J.F. \& Acrivos, A. 1982 The deformation and breakup of a slender drop in an extensional flow : inertial effects. J. Fluid Mech. 115, 443-451.

Gelier, A. S., Lee, S. H. \& Leal, L. G. 1986 The creeping motion of a spherical particle normal to a deformable interface. J. Fluid Mech. 169, 27-69.

Goedde, E. F. \& Yuen, M. C. 1970 Experiments on liquid jet instability. J. Fluid Mech. 40, 495-511.

Goren, S. L. 1964 The shape of a thread of liquid undergoing break-up. J. Colloid Sci. 19, $81-86$.

Grace, H. P. 1971 Dispersion phenomena in high viscosity immiscible fluid systems and application of static mixers as dispersion devices in such systems. Engng Found. Res. Conf. 3rd Andover, N.H. (Republished 1982 in Chem. Engng Commun. 14, 225-277.)

Grennspan, H. P. \& McCaY, B. M. 1981 On wetting of a surface by a very viscous fluid. Stud. Appl. Maths 64, 95-112.

Higdon, J. J. L. 1985 Stokes flow in arbitrary two-dimensional domains: shear flow over ridges and cavities. J. Fluid Mech. 159, 195-226.

KhakHaR, D. V. \& OtTino, J. M. 1987 Breakup of liquid threads in linear flows. Intl J. Multiphase Flow 13, 147-180. 
Lasheras, J. C., Fernandez-Pello, A. C. \& Dryer, F. L. 1979 Initial observations on the free droplet combustion characteristics of water-in-fuel emulsions. Combust. Sci. Tech, 21, 1-14.

LEE, S. H. \& LEAL, L. G. 1982 The motion of a sphere in the presence of a deformable interface. II. A numerical study of the translation of a sphere normal to an interface. J. Colloid Interface Sci. 87, 81-106.

LeE, W.-K. \& Flumerfelt, R.W. 1981 Instability of stationary and uniformly moving cylindrical fluid bodies - I. Newtonian systems. Intl J. Multiphase Flow 7, 363-384.

LeE, W.-K., YU, K.-L. \& Flumerfelt, R. W. 1981 Instability of stationary and uniformly moving cylindrical fluid bodies - II. Viscoelastic threads and experimental observations. Int J. Multiphase Flow 7, 385-400.

Mikami, T., Cox, R. G. \& Mason, S. G. 1975 Breakup of extending liquid threads. Intl J. Multiphase Flow 2, 113-138.

RALLison, J. M. 1981 A numerical study of the deformation and burst of a viscous drop in general shear flows. J. Fluid Mech. 109, 465-482.

Raluison, J. M. 1984 The deformation of small viscous drops and bubbles in shear flows. Ann. Rev. Fluid Mech. 16, 45 66.

Rallison, J. M. \& ACrivos, A. 1978 A numerical study of the deformation and burst of a viscous drop in an extensional flow. J. Fluid Mech. 89, 191-200.

RuMScheidt, F. D. \& Mason, S. G. 1962 Break-up of stationary liquid threads. J.Colloid Sci. 17, $260-269$.

Rutland, D. F. \& Jameson, G. J. 1971 A non-linear effect in the capillary instability of liquid jets. J. Fluid Mech. 46, 262-271.

SheRwood, J. D. 1988 Breakup of fluid droplets in electric and magnetic fields. J. Fluid Mech. 188, $133-146$.

Stone, H. A., Bentley, B. J. \& Leal, L. G. 1986 An experimental study of transient effects in the breakup of viscous drops. J. Fluid Mech. 173, 131-158.

TAYLOR, G. I. 1932 The viscosity of a fluid containing small drops of another fluid. Proc. R. Soc. Lond. A 138, 41-48.

TAYLOR, G. I. 1934 The formation of emulsions in definable fields of flow. Proc. R. Soc. Lond. A 146, 501-523.

TомотікA, S. 1935 On the instability of a cylindrical thread of a viscous liquid surrounded by another viscous fluid. Proc. $R$. Soc. Lond. A 150, 322-337.

TомотікA, S. 1936 Breaking up of a drop of viscous liquid immersed in another viscous fluid which is extending at a uniform rate. Proc. $R$. Soc. Lond. A 153, 302-318.

YoungReN, G. K. \& ACRIvos, A. 1975 Stokes flow past a particle of arbitrary shape; a numerical method of solution. J. Fluid Mech. 69, 377-403 (corrigendum 69, 813).

Youngren, G. K. \& ACrivos, A. 1976 On the shape of a gas bubble in a viscous extensional flow. J. Fluid Mech. 76, 433-442.

YUEN, M.-C. 1968 Non-linear capillary instability of a liquid jet. J. Fluid Mech. 33, 151-163. 